FEDERAL RESERVE BANK of ST. LOUIS

\section{RESEARCH DIVISION} Working Paper Series

\title{
Markov-Perfect Risk Sharing, Moral Hazard and Limited Commitment
}

\author{
Alexander K. Karaivanov \\ and \\ Fernando M. Martin
}

\author{
Working Paper 2011-030E \\ https://doi.org/10.20955/wp.2011.030
}

June 2018

\section{FEDERAL RESERVE BANK OF ST. LOUIS \\ Research Division \\ P.O. Box 442}

St. Louis, MO 63166

The views expressed are those of the individual authors and do not necessarily reflect official positions of the Federal Reserve Bank of St. Louis, the Federal Reserve System, or the Board of Governors.

Federal Reserve Bank of St. Louis Working Papers are preliminary materials circulated to stimulate discussion and critical comment. References in publications to Federal Reserve Bank of St. Louis Working Papers (other than an acknowledgment that the writer has had access to unpublished material) should be cleared with the author or authors. 


\title{
Markov-Perfect Risk Sharing, Moral Hazard and Limited Commitment*
}

\author{
Alexander K. Karaivanov \\ Simon Fraser University
}

\author{
Fernando M. Martin \\ Federal Reserve Bank of St. Louis
}

March 27, 2018

\begin{abstract}
We define, characterize and compute Markov-perfect risk-sharing contracts in a dynamic stochastic economy with endogenous asset accumulation and simultaneous limited commitment and moral hazard frictions. We prove that Markov-perfect insurance contracts preserve standard properties of optimal insurance with private information and are not more restrictive than a longterm contract with one-sided commitment. Markov-perfect contracts imply a determinate asset time-path and a non-degenerate long-run stationary wealth distribution. Quantitatively, we show that Markov-perfect risk-sharing contracts provide sizably more consumption smoothing relative to self-insurance and that the welfare gains from resolving the commitment friction are larger than the gains from resolving the moral hazard friction at low asset levels, while the opposite holds for high asset levels.
\end{abstract}

Keywords: Markov-perfect equilibrium, risk-sharing, limited commitment, moral hazard, consumption smoothing

JEL classification: D11, E21.

\footnotetext{
${ }^{*}$ We thank the editor, B. Ravikumar, two anonymous referees, A. Abraham, G. Camera, C. Carroll, M. Golosov, E. Green, N. Pavoni, V. Quadrini and audiences at CEF, Queen's, SED, Stony Brook and UC Santa Barbara for helpful comments and suggestions. Karaivanov acknowledges the financial support of the Social Sciences and Humanities Research Council of Canada. The views expressed in this paper do not necessarily reflect official positions of the Federal Reserve Bank of St. Louis, the Federal Reserve System, or the Board of Governors.
} 


\section{Introduction}

We define, characterize and compute Markov-perfect risk-sharing contracts in a dynamic economy with stochastic income and endogenous asset accumulation. We highlight the roles of two market frictions that simultaneously affect the degree of consumption and income smoothing: (i) limited commitment, by which we mean inability to commit to long-term contracts; and (ii) private information, in the form of moral hazard. Importantly, agents' wealth interacts with both frictions endogenously, as it affects their demand for insurance and their incentives and intertemporal trade-offs.

We define Markov-perfect insurance as a sequence of time-consistent and incentive-compatible one-period risk-sharing contracts, such that both agents and insurers cannot commit beyond the current period and which depend only on payoff-relevant variables. We characterize, theoretically and quantitatively, the properties of Markov-perfect risk-sharing contracts and compare them to two commonly studied alternatives: self-insurance and long-term contracts. Computationally, we also quantify the size and distribution of the welfare costs from the commitment and information frictions across agents with different wealth levels.

While there is a large literature on risk sharing subject to commitment and/or private information frictions, our motivation and approach differ in several important ways. First, in terms of motivation, the fundamental risk-sharing friction on which we focus is lack of long-term commitmentthe fact that in many situations individuals and firms cannot commit, or are legally not allowed, to enter binding long-term agreements (e.g., labor, rental, insurance, TV, phone, internet, etc.) Instead, both insurers and the insured can only commit short-term and can costlessly walk away at specified times. The Markov-perfect equilibrium (MPE) concept maps naturally into this setting.

Second, our Markovian approach to solving and characterizing the problem of dynamic insurance with commitment and information frictions does not use the mathematical abstraction of promised utility as a state variable. In fact, because of the lack of long-term commitment, making historycontingent promises about future consumption and using these promises to provide incentives and consumption smoothing over time is infeasible. Instead, the insurance transfers in an MPE can only depend on observable, payoff-relevant fundamentals (in our case, current output and wealth) which evolve endogenously over time with the history of income shocks.

Markov-perfect insurance restricts the contract space to recursive policy functions of current output and assets. However, we show that focusing on Markov-perfect contracts in our setting is not more restrictive than assuming one-sided commitment to an infinitely-long contract. Specifically, we prove an equivalence mapping between the MPE, in which the dynamic state variable is the agent's assets, and a setting with long-term commitment by the insurer, in which the state variable is promised utility. This equivalence obtains regardless of whether the agent's assets are contractible or not. In essence, the agent's assets "encode" the history of shocks and thus, completely determine the current and future terms of risk-sharing, just like promised utility in the standard approach. As we discuss in more detail below, these results relate, but also significantly differ from recent contributions by Albanesi and Sleet (2006) and Khan et al. (2014).

Importantly, our equivalence result can also be interpreted as a decomposition of a long-term contract in a setting with limited commitment and private information into a sequence of short-term insurance contracts. Such decomposition does not emerge in most dynamic contracting environments in which, as many authors going back to Townsend (1982) have shown, there are gains from enduring relationships. The key assumptions delivering the equivalence between MPE (sequence of 
short-term contracts) and a contract with long-term commitment are free entry and non-exclusivity in the insurance market and the agents' ability to carry assets over time.

We study an environment in which a risk-averse agent is endowed with a stochastic technology transforming labor effort into output. The agent can imperfectly self-insure through accumulating or drawing down a risk-free asset. A perfectly competitive risk-neutral insurer observes the agent's asset holdings, but does not observe the agent's effort, giving rise to a moral hazard problem.

We first show that Markov-perfect risk-sharing contracts with limited commitment and moral hazard provide partial insurance and are characterized by 'inverse Euler equations' relating the reciprocals of current and future marginal utility of consumption, therefore preserving the standard properties of optimal insurance with private information from the literature. Similarly, Markovperfect insurance contracts preserve the standard properties of optimal insurance with full information: consumption is equalized across states of the world and the relationship between present and future consumption is described by an Euler equation equalizing current marginal utility of consumption with appropriately discounted future marginal utility.

Assuming free entry in the insurance market, we prove and numerically verify that Markovperfect insurance contracts yield history-contingent time paths for consumption and effort which are equivalent to the time paths implied by an infinitely-long contract with full commitment by the insurer. The implications for asset dynamics of Markov-perfect insurance and insurance with onesided commitment are, however, very different. An insurer endowed with long-term commitment power uses utility promises to implement the desired risk-sharing allocation. This implies an indeterminacy in asset dynamics as the insurer can directly control the agent's consumption via current and future transfers, to which he is committed. In contrast, the inability of the insurer to commit long-term in our setting implies that the agent's asset holdings become an integral factor in risk sharing which yields non-trivial dynamics and non-degenerate limiting assets distribution that can be taken to data.

We use numerical methods to further characterize the properties of Markov-perfect insurance contracts. The MPE problem is tractable, low-dimensional and relatively easy to compute, both with and without private information. We show that an MPE can be parameterized to match several broad dimensions of US data. We find that Markov-perfect insurance contracts provide sizeable additional consumption smoothing relative to self-insurance, particularly for agents with low asset holdings. Even in the presence of very volatile output-shock realizations, access to Markov-perfect insurance allows agents to smooth consumption to a considerably higher degree compared to when only relying on their own savings. In addition, the transfers implemented by the Markov-perfect insurance contracts imply a much smoother income process for the agent.

The properties of Markov-perfect insurance described above have important consequences for wealth inequality. In an MPE a large fraction of agents have zero assets in the limiting distribution, since poorer agents have weaker incentive to supply effort and accumulate assets when they have access to outside insurance. Our model also delivers a long-term consumption distribution that is broadly in line with the data, avoiding the counterfactual degree of left skewness implied by models with limited commitment alone (see Broer et al., 2017). In addition, our approach allows us to compute the size and distribution over wealth of the welfare costs of moral hazard and limited commitment. For our parameterization, we find sizable gains from resolving either friction which are the highest at low-wealth levels. We also find that the gains from resolving the commitment friction are larger than the gains from resolving the moral hazard friction at low asset levels, while the opposite holds at high asset levels. 


\section{Related literature}

Our equivalence results between MPE and one-sided commitment contracts and between shortand long-term contracts complement but also significantly differ from two recent papers - Albanesi and Sleet (2006) and Khan et al. (2014). Albanesi and Sleet (2006), henceforth AS, study optimal taxation in a finite-horizon economy with private information due to unobserved i.i.d. labor disutility shocks. There are no commitment frictions and no real assets. Assuming separable preferences in consumption and labor effort and an exogenous lower bound on promised utility, the authors show that the constrained optimum in their private-information setting can be decentralized via a relatively simple system of non-linear taxes and non-contingent claims subject to borrowing constraints. Specifically, each agent's tax payment depends only on her current labor income and current claim holdings. The agents' claim holdings map to their promised utility value in the planner's problem and the tax schedule provides incentives for the "correct" sequence of claim holdings to be chosen in equilibrium.

Khan et al. (2014), henceforth KPR, study an infinite-horizon, linear-production economy with capital and private information due to unobserved productivity (output). The agent's capital holdings are observable and can be contracted upon. The authors characterize the constrained optimal allocation of consumption and capital and show that, assuming constant relative riskaversion (CRRA), the constrained optimum can be decentralized via consumption transfers and investment requirements that condition only on current capital. The agent's capital stock is shown to map one-to-one into promised utility in the planner's problem.

The common feature in these two papers and ours is the result that, under appropriate conditions, an equivalence mapping can be constructed between promised utility as the state variable in a mechanism design problem and another state variable in a different problem-non-contingent claims in AS, capital in KPR or assets in our paper. However, while the fundamental friction in both AS and KPR is private information with i.i.d. structure across agents and time, the fundamental friction in our setting is double-sided lack of long-term commitment. ${ }^{1}$ As is well-known from the literature, private information and commitment problems have very different natures and hence it is not obvious that there should exist a parallel in terms of results. Moreover, our equivalence result holds both with and without private information in the form of moral hazard (see Karaivanov and Martin, 2015).

Second, unlike KPR and AS who start from a planner's problem and decentralize it as a particular competitive equilibrium, we relate back to the literature on Markov-perfect equilibria by doing, in a sense, the opposite of AS and KPR - motivated by the lack of commitment assumption, we propose an MPE and show that, with free entry by insurers, it delivers equivalent allocations as a hypothetical long-term contract with one-sided commitment. In contrast, AS model a setting with full, double-sided commitment while in KPR there are no commitment problems since both sides do not have an incentive to renege at any time period. Third, beyond these major distinctions, there exist other potentially important differences between the three papers: private information vs. unobserved actions; finite (AS) vs. infinite horizon; endogenous (our paper) vs. exogenous (AS) utility lower bound; CRRA (KPR) vs. more general preferences, etc.

In another related recent paper Broer et al. (2017), henceforth BKK, study consumption risk sharing with unobservable persistent income shocks and limited enforcement (agents, but not insurers, can renege and go to autarky). The authors show that, in contrast to models with only limited enforcement, their model has implications about consumption similar to those in self-

\footnotetext{
${ }^{1}$ Output in our model is not i.i.d. over time since effort depends endogenously on assets.
} 
insurance (e.g., Aiyagari, 1994) and closer to the data. BKK also compare their model with the selfinsurance and limited enforcement settings in the context of a social insurance policy intervention. While our model also features both private information and limited commitment and delivers similar distribution of consumption, it differs from BKK in several important ways. We model two-sided lack of commitment and our information friction stems from an unobserved action which makes the income process endogenous. In contrast to BKK who use the conventional "promised utility" approach, we study Markov-perfect equilibria. This allows us to study asset accumulation dynamics and the long-run distribution of wealth, in addition to the model implications for consumption and income.

In Karaivanov and Martin (2015) we analyze Markov-perfect contracts with full information and exogenous income process and focus on the return-on-assets differential between agents and insurers with market power. We show that limited commitment by the insurer is restrictive and distorts the agent's consumption profile only when the insurer has a rate of return advantage and the agent is sufficiently wealthy. In contrast, in the current paper we assume away any return differential, endogenize the agent's effort and private information, and focus on the quantitative implications of Markov-perfect insurance contracts relative to other settings from the literature and data.

Our timing assumption regarding when parties can quit the contract differs from much of the literature on limited commitment (Thomas and Worrall, 1988, 1994; Kocherlakota, 1996; Ligon et al., 2002; and Krueger and Uhlig, 2006 among others). In those papers, the key issue is the potential inability to support full insurance when agents can opportunistically renege on the contract within a period, typically after high output is realized, and go to autarky or to another insurer. The result is that, under certain conditions, no additional consumption smoothing over self-insurance can be provided. In contrast, our agents and insurers can only quit at the beginning of a period, before output is realized and additional smoothing over self-insurance is always possible, including with two-sided lack of commitment. ${ }^{2}$ Furthermore, asset accumulation is not studied in those papers since the insurers are assumed to be able to commit to an infinitely-long contract and thus implement the incentive-feasible consumption allocation directly, through utility promises. In contrast, we emphasize the private asset dynamics that arise when both agents and insurers can commit within a period but not across periods.

In assuming two-sided lack of commitment our paper relates to Thomas and Worrall (1988), Kocherlakota (1996) and Ligon et al. (2002). Thomas and Worrall (1988) study a firm-worker relationship in which each party can always walk away to a competitive spot labor market. Kocherlakota (1996) and Ligon et al. (2002) study risk sharing among risk-averse households who can walk away to autarky, with the focus being on occasionally binding participation constraints and accounting for partial insurance, as observed in the data. In contrast, we model the lack of commitment problem as a Markov-perfect equilibrium and emphasize the role of asset accumulation in consumption smoothing.

Our paper also relates to a large literature emphasizing the gains from enduring relationships in settings with incomplete markets caused by private information or enforcement problems. ${ }^{3}$ Much of this literature assumes commitment to an infinitely-long agreement and does not allow agents to

\footnotetext{
${ }^{2}$ Our timing is similar to Phelan's (1995) who, however, assumes that insurers can fully commit, no asset accumulation and unobservable agent's income.

${ }^{3}$ Green (1987), Spear and Srivastava (1987), Thomas and Worrall (1990), Atkeson and Lucas (1992), Townsend (1982), Rogerson (1985b), Phelan and Townsend (1991) among many others.
} 
save or provide any other role for asset accumulation. ${ }^{4}$ While welfare improving ex-ante, the optimal long-term contracts exhibit counterfactual properties such as the agents' consumption converging to zero (immiseration), exploding consumption inequality, or degenerate long-run wealth distributions (see Phelan, 1998 for a review). In addition, long-term contracts can be time-inconsistent-at a later date a party may gain from reneging on the ex-ante agreement.

Our result on the allocation equivalence between Markov-perfect insurance contracts and a long-term contract with one-sided commitment by insurers relates to Malcomson and Spinnewyn (1988) and Fudenberg et al. (1990) who also analyze short- and long-term contracts in multiperiod moral hazard economies. ${ }^{5}$ While related, their results are not directly applicable to our setting for several reasons: (i) we study infinitely-long contracts; (ii) we do not assume two-sided commitment to the long-term contract; and (iii) with multiperiod moral hazard the Pareto frontier may not be downward sloping everywhere (Phelan and Townsend, 1991). Finally, Meh and Quadrini (2006) study a model of one-sided limited commitment via capital diversion, discuss a mapping from assets to promised utility and numerically verify the Fudenberg et al. (1990) conditions for their parameterization.

The paper is organized as follows. Section 2 describes the model environment. Section 3 defines and characterizes Markov-perfect insurance contracts and the roles played by private information and limited commitment. Section 4 explores numerically the properties of Markov-perfect contracts, compared to a self-insurance economy and US data. Section 5 quantifies the welfare costs of private information and limited commitment. Section 6 concludes. All proofs are in the Appendix.

\section{The Environment}

An infinitely-lived agent maximizes expected discounted utility over consumption $c$ and effort $e$. Period utility is given by $u(c)-e$, where $u_{c}(c)>0, u_{c c}(c)<0$ and $u$ satisfies Inada conditions. ${ }^{6}$ The agent discounts future utility by factor $\beta \in(0,1)$ and is endowed with stochastic production technology that maps effort into output. There are $n \geq 2$ possible output values, $y^{1}, \ldots, y^{n}$ where $0 \leq y^{1}<\ldots<y^{n}$. Denote by $\pi^{i}(e)$ the probability that output level $y^{i}$ is realized, given effort level $e$. Assume effort takes values on the set $\mathbb{E}$ which is a closed interval in $\mathbb{R}_{+}$.

Assumption 1 For all $e \in \mathbb{E}$ :

(i) $\sum_{i=1}^{n} \pi^{i}(e)=1$;

(ii) $\pi^{i}(e)>0$ for $i=1, \ldots, n$;

(iii) $\pi^{i}(e)$ are twice continuously differentiable for $i=1, \ldots, n$;

(iv) $\exists i=1, \ldots, n$ such that $\pi_{e}^{i}(e) \neq 0$;

\footnotetext{
${ }^{4}$ An exception is the work on hidden saving or borrowing, e.g. Allen (1985) or Cole and Kocherlakota (2001) from which we differ by assuming that the agent's asset accumulation is observable.

${ }^{5}$ Fudenberg et al. (1990) show that, under certain conditions on the information structure ruling out adverse selection, equal discount rates by the principal and the agent, downward sloping Pareto frontier and finite duration, a sequence of short-term contracts and a long-term contract yield equivalent allocations. Malcomson and Spinnewyn (1988) allow agents to save in an observable asset and prove equivalence between two consecutive one-period contracts and one two-period contract under certain conditions (see their Proposition 2).

${ }^{6}$ We use subscripts to denote partial derivatives and primes for next-period values. Assuming that period utility is linear in effort is without loss of generality given our assumptions on $\pi^{i}(e)$.
} 
(v) the monotone likelihood ratio property (MLRP) holds: $\frac{\pi_{e}^{i}(e)}{\pi^{i}(e)}$ is non-decreasing in $i$; and

(vi) the convexity of the distribution function condition (CDFC) holds, $\sum_{j=1}^{i} \pi_{e e}^{j} \geq 0$ for all $i=1, \ldots, n$.

Assumption 1 is relatively standard but some aspects deserve elaborating. Part (ii) is the familiar full support condition stating that any income level $y^{i}$ can be realized with positive probability from any feasible effort level, $e \in \mathbb{E}$. Part (iv) rules out the possibility that all probabilities $\pi^{i}$ do not depend on the agent's effort $e$ (in such case there would be no moral hazard problem when effort is unobservable). Parts (v) and (vi) are well-known sufficient conditions for the validity of the first-order approach in moral hazard models (Rogerson, 1985a).

The agent can save and borrow at the gross interest rate, $r$. Assume that the agent's asset holdings, $a$ belong to the bounded set $\mathbb{A}=[\underline{a}, \bar{a}]$.

Assumption 2 (i) $0<r<\beta^{-1}$; (ii) the lower bound on assets is the natural borrowing limit: $\underline{a}=-\frac{y^{1}}{r-1}$.

Assumption 2(i) is a standard condition ensuring finite long-run asset holdings. Assumption 2 (ii) sets the minimal asset level, $\underline{a}$ equal to the natural borrowing limit (Aiyagari, 1994) which allows us to focus on interior solutions for asset choices. We further assume that the upper bound $\bar{a}$ is sufficiently large so that it never binds in equilibrium.

\subsection{Self-insurance}

We first describe what the agent can achieve on his own, using savings to self-insure against output fluctuations. We use this self-insurance problem as an intuitive comparison benchmark in the theoretical and quantitative analysis of Markov-perfect risk sharing.

Consider an agent who can only self-insure by using assets as a buffer stock. Given current assets $a \in \mathbb{A}$, the timing of each period is as follows: (1) the agent decides how much effort $e$ to put in; (2) output $y^{i}$ is realized; and (3) the agent decides how much to consume, $c^{i}$ and how much assets to carry into the next period, $a^{i} \in \mathbb{A}$. The agent faces the state-by-state budget constraint, $c^{i}+a^{i}=r a+y^{i}$. The agent's self-insurance problem can be written recursively as

$$
\Omega(a)=\max _{e,\left\{a^{i}\right\}_{i=1}^{n}} \sum_{i=1}^{n} \pi^{i}(e)\left[u\left(r a+y^{i}-a^{i}\right)+\beta \Omega\left(a^{i}\right)\right]-e,
$$

where $\Omega(a)$ denotes the agent's self-insurance value function. ${ }^{7}$

It is easy to show that $\Omega(a)$ is strictly increasing in agent's assets. Intuitively, since $r \beta<1$ by Assumption 2, the agent only saves to insure against future consumption volatility. Clearly, an agent with more assets can do everything an agent with less assets can, but is in a better position to self-insure against a sequence of low output realizations.

As is standard in such models, since $n \geq 2$ states cannot be spanned using a single asset, consumption smoothing is imperfect $\left(c^{i}\right.$ differs across states with different $\left.y^{i}\right)$. Other easy-to-show

\footnotetext{
${ }^{7}$ Using standard arguments, our assumptions on $u$, together with $r \beta<1$ and the upper bound on assets $\bar{a}$ are sufficient for the self-insurance problem (SI) to be well-defined (details available upon request).
} 
properties of self-insurance are that the optimal consumption, $c^{i}$ and next-period assets, $a^{i}$ in each state are increasing in current assets $a$. Asset holdings are reduced if the lowest output state(s) are realized and increased (for some asset range) if the highest state(s) are realized. ${ }^{8}$

\section{Markov-Perfect Insurance}

\subsection{Markov-perfect equilibrium}

Suppose now the agent has access to a perfectly competitive insurance market with free entry populated by risk-neutral profit-maximizing insurers. Demand for market insurance exists in our setting since the agent cannot span the $n$-dimensional output uncertainty by borrowing and saving in the single non-contingent asset $a$.

We assume limited commitment in the form that neither insurers nor agents can commit to a contract extending beyond the current period. One-period risk-sharing contracts are, however, perfectly enforceable. Our notion of limited commitment thus differs from other papers in the literature in which contract parties can opportunistically renege within a period. The agent's effort is not observable by the insurers (private information). In contrast, the agent's assets, $a$ are always observable and the choice of the next-period asset level (which occurs after output is realized) is assumed contractible. ${ }^{9}$

We study insurance contracts the terms of which depend only on fundamentals, that is, payoffrelevant variables: the agent's beginning-of-period assets, $a$ and the current output (state) realization, $y^{i}$. Following Maskin and Tirole (2001), we call these contracts Markov-perfect insurance contracts. Specifically, an insurance contract is offered before effort is exerted and consists of output-contingent transfers, $\tau^{i}$ and end-of-period asset holdings, $a^{i}$, both as functions of the agent's beginning-of-period assets, $a \in \mathbb{A}$. Agents with different beginning-of-period assets are, in general, offered different contracts. The insurer takes into account the agent's incentives to exert effort given the contract terms via an incentive-compatibility constraint. Since the insurer cannot commit beyond the current period, he takes future insurance contracts as given. That is, the insurer takes as given the agent's present value utility $\mathcal{V}\left(a^{i}\right)$, to be defined formally below, induced by the contracts implemented in the future which themselves depend on the agent's assets. The insurer can affect the agent's continuation value $\mathcal{V}\left(a^{i}\right)$ through the contractible asset choice, $a^{i}$. In equilibrium, the Markov-perfect insurance contracts thus consist of policy functions for current transfers and future asset holdings, as functions of beginning-of-period assets and output realization: $\left\{\mathcal{T}^{i}(a), \mathcal{A}^{i}(a)\right\}_{i=1}^{n}$.

After entering a contract with an insurer, the agent chooses an effort level, taking as given the current and anticipated future contracts delivering the value $\mathcal{V}\left(\mathcal{A}^{i}(a)\right)$. Call the associated state-contingent agent consumption $\mathcal{C}^{i}(a) \equiv r a+\mathcal{T}^{i}(a)-\mathcal{A}^{i}(a)$. The agent's effort choice problem is

$$
\max _{e} \sum_{i=1}^{n} \pi^{i}(e)\left[u\left(\mathcal{C}^{i}(a)\right)+\beta \mathcal{V}\left(\mathcal{A}^{i}(a)\right)\right]-e,
$$

\footnotetext{
${ }^{8}$ The proofs of these statements are standard and available upon request.

${ }^{9}$ See Karaivanov and Martin (2016) for an analysis of asset non-contractibility in a related dynamic setting without a moral hazard problem.
} 
with first-order condition,

$$
\sum_{i=1}^{n} \pi_{e}^{i}(e)\left[u\left(\mathcal{C}^{i}(a)\right)+\beta \mathcal{V}\left(\mathcal{A}^{i}(a)\right)\right]-1=0 .
$$

The insurer must take into account how the agent's effort responds to the offered contract (incentive-compatibility). We use the "first-order approach" (Rogerson, 1985a) and impose the agent's first-order condition for effort choice, (1) as a constraint in the contracting problem. By Assumption 1, the output probabilities $\pi^{i}(e)$ satisfy sufficient conditions for the validity of the first-order approach: the monotone likelihood ratio property (MLRP) and the convexity of the distribution function condition (CDFC) - see Rogerson (1985a). Alternative sufficient conditions to parts (v) and (vi) of Assumption 1 are also possible. ${ }^{10}$

Free entry in the insurance market results in zero expected profits in each period and each sub-market indexed by agent's asset holdings, $a$. Cross-subsidization across agents with different asset levels is ruled out - if an insurer makes profits on agents with some asset levels but a loss on others, another insurer can offer a better contract to the former (assets are observable). Perfect competition also implies that all the surplus from risk sharing goes to the agent. The problem of an insurer facing an agent with assets $a \in \mathbb{A}$ can be thus written as

$$
\max _{e,\left\{\tau^{i}, a^{i}\right\}_{i=1}^{n}} \sum_{i=1}^{n} \pi^{i}(e)\left[u\left(c^{i}\right)+\beta \mathcal{V}\left(a^{i}\right)\right]-e
$$

subject to incentive-compatibility and zero per-period profits for the insurer:

$$
\begin{aligned}
\sum_{i=1}^{n} \pi_{e}^{i}(e)\left[u\left(c^{i}\right)+\beta \mathcal{V}\left(a^{i}\right)\right]-1 & =0 \\
\sum_{i=1}^{n} \pi^{i}(e)\left[y^{i}-\tau^{i}\right] & =0,
\end{aligned}
$$

where we use $\left\{\tau^{i}, a^{i}\right\}_{i=1}^{n}$ for the current-period contract and define $c^{i} \equiv r a+\tau^{i}-a^{i}$ to simplify the notation.

Next, we formally define a Markov-perfect equilibrium and Markov-perfect insurance contracts.

Definition 1 A Markov-perfect equilibrium (MPE) is a set of functions $\left\{\mathcal{E},\left\{\mathcal{T}^{i}, \mathcal{A}^{i}\right\}_{i=1}^{n}, \mathcal{V}\right\}$ : $\mathbb{A} \rightarrow \mathbb{E} \times \mathbb{R}^{n} \times \mathbb{A}^{n} \times \mathbb{R}$ such that, $\forall a \in \mathbb{A}$ :

$$
\left\{\mathcal{E}(a),\left\{\mathcal{T}^{i}(a), \mathcal{A}^{i}(a)\right\}_{i=1}^{n}\right\}=\underset{e,\left\{\tau^{i}, a^{i}\right\}_{i=1}^{n}}{\operatorname{argmax}} \sum_{i=1}^{n} \pi^{i}(e)\left[u\left(c^{i}\right)+\beta \mathcal{V}\left(a^{i}\right)\right]-e
$$

subject to (IC) and (ZP) and where

$$
\mathcal{V}(a)=\sum_{i=1}^{n} \pi^{i}(\mathcal{E}(a))\left[u\left(\mathcal{C}^{i}(a)\right)+\beta \mathcal{V}\left(\mathcal{A}^{i}(a)\right)\right]-\mathcal{E}(a),
$$

with $\mathcal{C}^{i}(a) \equiv r a+\mathcal{T}^{i}(a)-\mathcal{A}^{i}(a)$ and $c^{i}=r a+\tau^{i}-a^{i}$.

A Markov-perfect insurance contract for any asset level $a \in \mathbb{A}$ consists of the statecontingent transfers and end-of-period asset choices $\left\{\tau^{i}=\mathcal{T}^{i}(a), a^{i}=\mathcal{A}^{i}(a)\right\}_{i=1}^{n}$ associated with an $M P E$.

\footnotetext{
${ }^{10}$ For example, if $n=2$ it is easy to verify directly that it is sufficient to assume that $\pi^{2}(e)$ is strictly increasing and strictly concave in $e$.
} 


\subsection{Characterization}

We now characterize the properties of Markov-perfect insurance contracts. First, note that the constraint set of the problem in Definition 1 is non-empty for all $a \in \mathbb{A}$. For example, a fullinsurance (equal consumption) contract with $e=\min \{\mathbb{E}\}$, or the contract setting $\tau^{i}=y^{i}$ and $\left\{e, a^{i}\right\}$ to their self-insurance values (see problem SI) are feasible and incentive-compatible. Since the set $\mathbb{A}$ is compact, existence of a fixed point $\mathcal{V}$ in problem (2)-(ZP) can be shown as in Abraham and Pavoni (2008) using standard contraction mapping arguments.

Second, for any $a \in \mathbb{A}$, it is easy to see that Markov-perfect insurance yields larger present value utility than self-insurance. That is, $\mathcal{V}(a) \geq \Omega(a)$, where the self-insurance value function $\Omega(a)$ was defined in (SI). In all our numerical simulations this inequality is always strict. Intuitively, the selfinsurance allocation is always feasible (satisfies constraints IC and ZP) but not necessarily optimal for the Markov-perfect insurance problem. Thus, with free entry, an agent can never be worse-off in an MPE compared to in self-insurance. In other words, in an MPE there are gains from risk sharing over and above self-insurance. We characterize and quantify these gains in more detail in Section 4 .

Proposition 1 Given Assumptions 1 and 2, an MPE is characterized by:

(i) monotonicity: consumption, $\mathcal{C}^{i}(a)$ is non-decreasing in $i$;

(ii) partial insurance: $\mathcal{C}^{1}(a)<\mathcal{C}^{n}(a)$ for all $a \in \mathbb{A}$; and $\mathcal{C}^{i}(a)<\mathcal{C}^{j}(a)$ for all $a \in \mathbb{A}$ and $1 \leq i<$ $j \leq n$ if and only if $\frac{\pi_{e}^{i}(e)}{\pi^{i}(e)}<\frac{\pi_{e}^{j}(e)}{\pi^{j}(e)} ;$

(iii) inverse Euler equations:

$$
\frac{1}{u_{c}\left(\mathcal{C}^{i}(a)\right)}=\frac{1}{\beta r} E\left[\frac{1}{u_{c}\left(\mathcal{C}\left(\mathcal{A}^{i}(a)\right)\right)}\right], \forall i \text { and } \forall a \in \mathbb{A} .
$$

(iv) irrelevance of asset contractibility: whether the agent's assets are contractible or non-contractible does not affect the Markov-perfect insurance contract $\forall a \in \mathbb{A}$.

Proposition 1 shows that Markov-perfect insurance contracts preserve standard features of optimal insurance with private information. Part (i) shows that higher output realizations imply (weakly) higher consumption. Parts (ii) and (iii) show that Markov-perfect contracts do not provide full insurance and the MPE consumption time path is characterized by the familiar inverse Euler equations from the literature on multi-period moral hazard with commitment (e.g., Rogerson, 1985b; Golosov et al., 2006).

Part (iv) of Proposition 1 demonstrates that whether the agent's asset holdings are contractible (or, directly controlled by the insurer) or non-contractible (freely chosen by the agent) results in equivalent MPE. That is, given the transfers in an MPE, for any current observable assets $a \in \mathbb{A}$, agents have no incentive to choose a future assets level $a^{\prime}$ that differs from the value $\mathcal{A}(a)$ specified by the contract. The key reason for this result is the free-entry assumption - since all the surplus goes to the agent, there is no misalignment of dynamic incentives between the insurer and insured. 


\subsection{The role of private information}

To illustrate the role of private information in Markov-perfect insurance, we compare the MPE characterized above with an analogous setting in which the agent's effort is observable and contractible. An MPE with full information is defined as in Definition 1 but without imposing constraint (IC). Let $\bar{y}(e)=\sum_{i=1}^{n} \pi^{i}(e) y^{i}$ denote expected output given effort $e \in \mathbb{E}$.

Proposition 2 An MPE with full information (observable effort) has the following properties:

(i) full insurance: $c^{i}=c^{j}=c=\mathcal{C}(a) ; a^{i}=a^{j}=a^{\prime}=\mathcal{A}(a)$ and $\tau^{i}=\bar{y}(e)$ for all $i, j=1, \ldots, n$ and $\forall a \in \mathbb{A}$

(ii) standard Euler equation:

$$
u_{c}(\mathcal{C}(a))=\beta r u_{c}(\mathcal{C}(\mathcal{A}(a)))
$$

(iii) consumption: strictly decreasing over time, $\mathcal{C}(a)>\mathcal{C}(\mathcal{A}(a))$; with $\mathcal{C}(a)$ strictly increasing $\forall a \in \operatorname{int} \mathbb{A}$

(iv) assets: strictly decreasing over time, $\mathcal{A}(a)<a$; with $\mathcal{A}(a)$ strictly increasing $\forall a \in$ int $\mathbb{A}$

(v) effort: $\mathcal{E}(a)$ decreasing in a.

When the agent's effort is observable and contractible, Markov-perfect insurance contracts yield standard properties of risk sharing with full information. Consumption is equalized across output states, since there is no insurance-incentives trade-off. Unlike in Thomas and Worrall (1988) or many others who define limited commitment as the inability to prevent an agent to opportunistically renege on the contract after a high-output realization, our limited commitment friction does not distort consumption within the period. In contrast to Proposition 1, a standard non-distorted Euler equation connecting current and future consumption via a decreasing time profile $($ since $\beta r<1$ ) is satisfied because of the removed need to provide incentives to put effort. In addition, with free entry, the insurer's and agent's asset accumulation incentives are aligned. The agent's effort decreases in assets since the marginal return on effort is lower for wealthier agents who finance a larger part of their consumption with the asset returns, $\mathrm{ra}$.

\subsection{The role of limited commitment}

We next study the role of limited commitment while keeping the private information friction (unobserved effort). To do so we compare the Markov-perfect insurance contracts with the optimal risk-sharing contract in two alternative settings in which long-term commitment is assumed possible. Specifically, we call full-commitment (two-sided commitment) the setting in which both the agent and the insurer can commit to an infinitely-lived contract at time zero. We call one-sided commitment the setting in which only the insurer can commit to a long-term contract. In this latter case, the agent can still walk away from a contract at the beginning of each period, as in our MPE. To simplify the exposition and for analytical tractability, assume from now on that the agent cannot borrow.

Assumption 3 The agent cannot borrow, that is, the lower bound on assets is zero: $\underline{a}=0$. 
Assume also that the insurer discounts future profits with factor $1 / r$, that is, he has the same intertemporal return as the agent. We can write the dynamic insurance problem with (full or one-sided) commitment as a two-stage problem. In the first stage, without loss of generality, the insurer extracts the agent's initial assets $a_{0}$ (that is, sets $a_{t}^{i}=0$ for all $t>0$ and $i=1, \ldots, n$ ) and promises in exchange present-value utility, $w_{1}^{i}$ from the next period onward. ${ }^{11}$ When commitment is one-sided (by the insurer only), the problem is also subject to the agent's limited-commitment constraint, $w_{1}^{i} \geq \underline{w}$ for all $i$, where $\underline{w}$ is the lowest feasible present-value utility for which the agent would not quit. In the second-stage problem, after the asset extraction, optimal consumption $c^{i}$ and promised utility $w^{i}$ are chosen respecting promise keeping (and, if one-sided commitment, $w^{i} \geq \underline{w}$ ). See the Appendix for full details and definitions.

Let $s_{t} \in\{1, \ldots, n\}$ be the output state in period $t$ and let $s^{t} \equiv\left\{s_{0}, \ldots, s_{t}\right\}$ denote the history of output states from period 0 up to period $t$. For any initial agent assets $a_{0} \in \mathbb{A}$, the constrained-optimal insurance contract with long-term commitment implies history-contingent sequences for consumption (equivalently transfers) and recommended effort. Denote these sequences as $\left\{c\left(a_{0}, s^{t}\right), e\left(a_{0}, s^{t}\right)\right\}_{t=0}^{\infty}$. For any $t=0, \ldots, \infty$ and any history $s^{t}$, call $\alpha\left(s^{t-1}\right)$ the beginning-ofperiod asset holdings by the agent, obtained by applying the policy rule in the Markov-perfect insurance setting, $\mathcal{A}^{i}(a)$. That is, call $\alpha\left(s^{t}\right)=\mathcal{A}^{s_{t}}\left(\alpha\left(s^{t-1}\right)\right)$ with $\alpha\left(s^{-1}\right)=a_{0}$. We prove the following equivalence result.

Proposition 3 For any initial assets $a_{0} \in \mathbb{A}$ and any output state history $s^{T}, T=1,2, \ldots \infty$, the optimal contract with one-sided commitment by the insurer and promised utility set $W^{M P} \equiv$ $[\mathcal{V}(0), \mathcal{V}(\bar{a})]$ yields history-contingent consumption and effort sequences $\left\{c\left(a_{0}, s^{t}\right), e\left(a_{0}, s^{t}\right)\right\}_{t=0}^{T}$ identical to the sequences $\left\{\mathcal{C}^{s t}\left(\alpha\left(s^{t-1}\right), \mathcal{E}\left(\alpha\left(s^{t-1}\right)\right)\right\}_{t=0}^{T}\right.$ generated by the MPE defined in $(2)-(\mathrm{ZP})$. In addition, $\mathcal{V}^{C}(a)=\mathcal{V}(a)$ for all $a \in \mathbb{A}$ where $\mathcal{V}^{C}(a)$ is the agent's value function in the one-sided commitment contract.

Proposition 3 shows that, under appropriate conditions on the set of promised utilities, the consumption and effort allocations in MPE are equivalent to the corresponding optimal allocations with long-term commitment by the insurer, for any history starting from any initial assets $a_{0} \in$ $\mathbb{A}$ (see the Appendix for full details on the one-sided commitment problem). That is, Markovperfect insurance contracts are not more restrictive than dynamic insurance contracts with one-sided commitment which have been studied by many authors. However, there is an important difference between the Markov-perfect and one-sided commitment settings. The insurance contract with onesided commitment has an indeterminate path for the agent's assets, since assets and promised utility are interchangeable in implementing future allocations (see the proof of Proposition 3 in the Appendix). In contrast, in an MPE the insurer cannot use promised utility (cannot commit to future transfers) as he lacks the ability to commit beyond the present period. This means that the agent's asset holdings are the only instrument which insurers can use to affect the contract's future value. Importantly, this leads to determinate and non-degenerate asset (savings) dynamics which can be used to evaluate the MPE model numerically and empirically, as we show in Sections 4 and 5 .

The equivalence result in Proposition 3 requires: (i) a lower bound on promised utility in the one-sided commitment contract equal to the MPE value at zero assets, $\underline{w}=\mathcal{V}(0)$; and (ii) that all utility promises are bounded from above by $\mathcal{V}(\bar{a})$. Since with one-sided commitment the equilibrium

\footnotetext{
${ }^{11}$ In Karaivanov and Martin (2015) we formally show this result in a full-information setting. It is straightforward to adapt the proof for the setting here.
} 
lower bound $\underline{w}$ equals the agent's outside option value (the lowest promised utility for which the agent would not quit), we can interpret the one-sided commitment contract in Proposition 3 as a long-term risk-sharing contract which the agent can quit at the beginning of each period and go to a Markov-perfect insurer, with zero assets. However, since we show that $\mathcal{V}^{C}(0)=\mathcal{V}(0)$, the outside option is also equivalent to the agent going to another insurer with one-sided commitment. The interpretation of the outside option is consistent with our assumption of free entry by insurers (no exclusive contracts).

Regarding the upper bound of the set $W^{M P}$ in Proposition 3, there is no general guarantee that promised utility in the one-sided commitment setting may not exceed a particular fixed value after some output history. ${ }^{12}$ For the case of full information and exogenous probabilities $\pi^{i}$, in Karaivanov and Martin (2015) we prove that promised utility in a one-sided commitment setting is strictly decreasing over time when $\beta r<1$. Hence, whenever the initial promise in the one-sided commitment setting satisfies $w_{0} \in[\mathcal{V}(0), \mathcal{V}(\bar{a})]$, the upper bound $\mathcal{V}(\bar{a})$ is never exceeded by the future promised utility choices (in fact, $w_{0}$ is never exceeded), for any history. While we do not have a formal proof of this result, we conjecture that, for a sufficiently large upper bound $\bar{a}$ on assets, the same property (promised utility choices bounded from above) also holds in our current MPE setting with private information. We check and confirm this conjecture numerically in Section 4. Specifically, for our choice of $\bar{a}$, we verify that for sufficiently high current promised utility levels $w \in[\mathcal{V}(0), \mathcal{V}(\bar{a})]$, the next-period promised utility choices, $w^{i}$ are strictly lower for any output level; that is, $w^{i}<w$ for all $i=1, \ldots, n$.

To help further understand Proposition 3, note that its equivalence result would not hold if promising present-value utility smaller than $\mathcal{V}(0)$ were feasible for the insurer, or if $\bar{a}$ were such that promised utility choices larger that $\mathcal{V}(\bar{a})$ were optimal. For example, in a setting with twosided commitment by both agents and insurers (which we compute in Section 5), promised utility values smaller than the lowest value in an MPE, $\mathcal{V}(0)$ are feasible, as the agent cannot quit the contract ex-post. ${ }^{13}$

\section{Quantitative Analysis}

In this section, we use numerical methods to further describe the properties of Markov-perfect insurance contracts with private information and limited commitment. We use the self-insurance economy without borrowing as a comparison benchmark. We compute and describe the Markovperfect insurance policy functions and sample time-paths as well as its implications for the degree of risk sharing and the long-run stationary distributions of wealth and consumption. We perform these exercises using a parameterization chosen to match aggregate features of the US data as in Castañeda et al. (2003). In Section 5 we then use the parameterized model to quantify the welfare gains from eliminating either the private information or the commitment frictions and show that their relative severity is heterogeneous and depends on the agent's wealth.

\footnotetext{
${ }^{12}$ For example, under moral hazard, large values of promised utility may be needed to provide incentives after long history of high output realizations and are feasible, since with commitment the insurer can run a loss in a given period.

${ }^{13}$ Indeed, if $u(0)=-\infty$ then promised utility values $w^{i} \rightarrow-\infty$ are optimally used to provide incentives, as in the standard immiseration result from the literature on multi-period moral hazard.
} 


\subsection{Parameterization}

We assume a generalized constant-relative-risk-aversion (CRRA) utility function,

$$
u(c)=\frac{\alpha\left(c^{1-\sigma}-1\right)}{1-\sigma}
$$

where $\alpha>0$ and $\sigma>0$. There are three possible output levels, "low", "medium" and "high", labeled $y^{L}, y^{M}$ and $y^{H}$ respectively. The probability functions, $\pi^{i}(e)$ for $i=\{L, M, H\}$ are

$$
\begin{aligned}
\pi^{L}(e) & =1-\pi^{M}(e)-\pi^{H}(e) \\
\pi^{M}(e) & =\frac{\varphi e^{\nu}}{1+e^{\nu}} \\
\pi^{H}(e) & =\frac{(1-\varphi) e^{\nu}}{\gamma+e^{\nu}},
\end{aligned}
$$

where $\nu>0, \gamma>1$ and $\varphi \in(0,1)$. It is easy to verify that $\pi^{i}(e)$ satisfy the sufficient conditions in Assumption 1.

To parameterize the MPE and self-insurance models we follow Castañeda et al. (2003) who match earnings, the wealth distribution and other aggregates for the US economy. Table 1 displays the resulting parameters. For the three output levels, $y^{L}, y^{M}$ and $y^{H}$, we use the Castañeda et al. (2003) values for the endowments of labor efficiency units. ${ }^{14}$ We set $\nu$ arbitrarily to 0.5 and pick the output probability distribution parameters $\varphi$ and $\gamma$ so that the simulated long-run distribution of realized output levels matches the corresponding proportions in the US data reported in Castañeda et al. (2003)- see Table 2.

Table 1: Parameter values

\begin{tabular}{|c|c|c|c|c|c|c|c|c|c|}
\hline$\alpha$ & $\beta$ & $\sigma$ & $r$ & $\nu$ & $\varphi$ & $\gamma$ & $y^{L}$ & $y^{M}$ & $y^{H}$ \\
\hline 4.000 & 0.924 & 1.500 & 1.061 & 0.500 & 0.450 & 2.000 & 0.100 & 0.315 & 0.978 \\
\hline
\end{tabular}

The values for the discount factor $\beta$ and the risk aversion parameter $\sigma$ are taken directly from Castañeda et al. (2003). We also set $r$ equal to the value implied by their calibration for the annual interest rate net of depreciation. The final free parameter, $\alpha$ only affects the scale of effort and we set it sufficiently large so that effort is significantly different from zero for all asset levels.

Following Castañeda et al. (2003) and the related literature, we assume that the agents are subject to a non-borrowing constraint, that is, $\underline{a}=0$. This implies that we can interpret the selfinsurance setting defined in Section 2 as a storage economy. In our parameterization the constraint $\underline{a}=0$ binds only when output is at its lowest state $\left(y^{L}\right)$ and assets are close to zero.

Table 2 shows the fractions of agents with each output realization in the long-run stationary distributions in MPE, self-insurance and the data. As explained above, the MPE values were targeted to match the reported fractions in Castañeda et al. (2003). However, the corresponding values in our self-insurance economy are also very similar. This suggests that the differences between

\footnotetext{
${ }^{14}$ See Table 5 in Castañeda et al. (2003). Note that they parameterize four endowment levels; the fourth type is about 1,000 times more productive than the first type and comprises $0.04 \%$ of working-age households. Since our economy already makes important simplifications with respect to theirs - no life-cycle features, taxes, etc. - we omit this fourth type to simplify the numerical analysis and exposition of results.
} 
the MPE and self-insurance economies described below do not stem from targeting these specific moments of the data within the MPE setting but are fundamentally related to the additional insurance provided by the Markov-perfect contracts.

Table 2: Long-run measure of agents, according to output realizations

\begin{tabular}{|l|c|c|c|}
\hline & $y^{L}$ & $y^{M}$ & $y^{H}$ \\
\hline Self-insurance (storage) & 0.59 & 0.23 & 0.19 \\
MPE & 0.61 & 0.22 & 0.18 \\
Castañeda et al. (2003) & 0.61 & 0.22 & 0.17 \\
\hline
\end{tabular}

Using the 1998 Survey of Consumer Finances, Budría-Rodríguez et al. (2002) report bivariate correlations between earnings and income, earnings and wealth, and income and wealth in the US equal to $0.72,0.47$ and 0.60 , respectively. Although we do not explicitly target these moments, our simulated economy is close to the US data along this dimension. Specifically, in the MPE, defining income as transfers plus capital income, $\tau^{i}+(r-1) a$; defining earnings as output, $y^{i}$ for $i=\{L, M, H\}$; and defining wealth as assets $a$, we obtain bivariate correlations between earnings and income, earnings and wealth and income and wealth of $0.81,0.39$ and 0.67 respectively.

While our theoretical framework emphasizes the role of moral hazard and limited commitment in a dynamic setting with endogenous labor effort and asset accumulation, it abstracts from other realistic elements modeled in the literature, notably a more detailed structure of the income process - for example, including both permanent and transitory components (e.g., Broer et al., 2017). ${ }^{15}$ Nevertheless, in Section 4.3.2 we show that MPE insurance contracts successfully approximates several features of those alternative models and/or the data, as related to the degree of consumption smoothing and the long-run distribution of wealth, despite not targeting these features directly.

\subsection{Computation}

We first compute the self-insurance (storage) model. We use a 1,000-point discrete grid for the state space $\mathbb{A}=[0, \bar{a}]$ but allow all choice variables to take any admissible value. ${ }^{16}$ We use the same assets grid for all computations performed below. Cubic splines are used to interpolate between the grid points. The upper bound for assets, $\bar{a}$ is set to 60 which ensures that all three asset policy functions, $\mathcal{A}^{i}(a)$ cross the 45-degree line. For clarity of exposition, all graphs below only display asset holdings up to $a=5$, which includes $99.95 \%$ of agents in a stationary equilibrium in the self-insurance economy and virtually all agents in MPE.

To compute the Markov-perfect equilibrium described in Definition 1, we use the following iterative algorithm to find the fixed-point in the value function $\mathcal{V}(a)$ and the policy functions: (i) start with the agent's value in the storage economy as an initial guess for $\mathcal{V}(a)$; (ii) solve the insurer's problem (2)-(ZP) which outputs a new value function; (iii) update and continue iterating until convergence. Subsequently, we use the first-order conditions of the insurer's problem to improve

\footnotetext{
${ }^{15}$ Note that our model does feature a degree of endogenous persistence in the earnings process via the effort choice which is a function of assets.

${ }^{16}$ We did not find significant gains from further increasing the size of the $\mathbb{A}$ grid. For example, the value function at $a=0$ computed with 1,000 vs. 10,000 grid points differs by only $0.02 \%$.
} 
the precision of the solution. Finally, we compute the long-run stationary distribution of assets by assuming a continuum of agents. This is done using standard techniques: the decisions rules derived from the numerical solution imply a transition matrix on which we iterate until obtaining a distribution that maps into itself.

\subsection{Markov-perfect insurance vs. self-insurance}

\subsubsection{Consumption smoothing}

To provide further insights into the workings of Markov-perfect insurance contracts we first describe their implications for consumption smoothing and compare these implications to those in the selfinsurance model with endogenous effort choice (SI). Consider an agent with assets $a \in \mathbb{A}$ and output realization $y^{i}, i=\{L, H, M\}$ and define the agent's income in MPE $\left(m^{i}\right)$ and in the self-insurance economy $\left(\hat{m}^{i}\right)$ respectively as,

$$
m^{i}=\tau^{i}+(r-1) a \quad \text { and } \quad \hat{m}^{i}=y^{i}+(r-1) a .
$$

We can then write the agent's consumption in MPE and self-insurance respectively as:

$$
\begin{aligned}
& c^{i}=\tau^{i}+r a-a^{i}=m^{i}-\left(a^{i}-a\right) \\
& \hat{c}^{i}=y^{i}+r a-\hat{a}^{i}=\hat{m}^{i}-\left(\hat{a}^{i}-a\right)
\end{aligned}
$$

where $\tau^{i}=\mathcal{T}^{i}(a)$ and $a^{i}=\mathcal{A}^{i}(a)$ in the MPE, and where $\hat{a}^{i}$ is the optimal end-of-period asset choice in the self-insurance economy. The expressions $a^{i}-a$ and $\hat{a}^{i}-a$ are the agent's change in assets (net savings).

The decomposition in (4)-(5) helps clarify the mechanism of consumption smoothing in the MPE vs. self-insurance (SI) settings. The output realizations $y^{i}$ enter consumption directly in selfinsurance and can be smoothed only by accumulating or decreasing the non-contingent asset stock. That is, smoothing in the self-insurance setting is only possible across time, by borrowing from the future in bad times and saving in good times. In contrast, the endogenous insurance transfers $\tau^{i}$ in MPE enable both smoothing across time periods, as in self-insurance, but, in addition, smoothing across states of the world within any given period (as if "borrowing" from high-income states). In other words, Markov-perfect insurance allows both (i) income smoothing (note that $m^{i}$ does not vary one-to-one with the output realization $y^{i}$ ) and (ii) further consumption smoothing, out of the already smoothed income. In contrast, in the self-insurance setting the only mechanism to smooth consumption in response to output shocks is via assets.

Figure 1 illustrates the consumption smoothing in MPE vs. the self-insurance economy. The upper left panel plots the Markov-perfect insurance transfers, $\tau^{i}=\mathcal{T}^{i}(a)$ against output, showing the extent to which the agent and the insurer share risk via smoothing the agent's income compared to self-insurance. If realized output is low $\left(y^{L}\right)$ the insurer provides the agent with higher non-asset income component than under self-insurance, that is, $\tau^{L}>y^{L}$ in (3), while, if realized output is medium $\left(y^{M}\right)$ or high $\left(y^{H}\right)$, the opposite holds.

The upper right panel of Figure 1 displays the agent's net savings in the MPE and the selfinsurance economies. For the lowest income state net savings as function of the agent's assets $a$ are nearly identical in both settings. In contrast, for the highest output state savings are much larger when the agent is self-insuring. Intuitively, in the self-insurance setting the agent chooses to stock up assets for future "bad times". This asset accumulation incentive is dampened in the MPE since 
Figure 1: Policy Functions
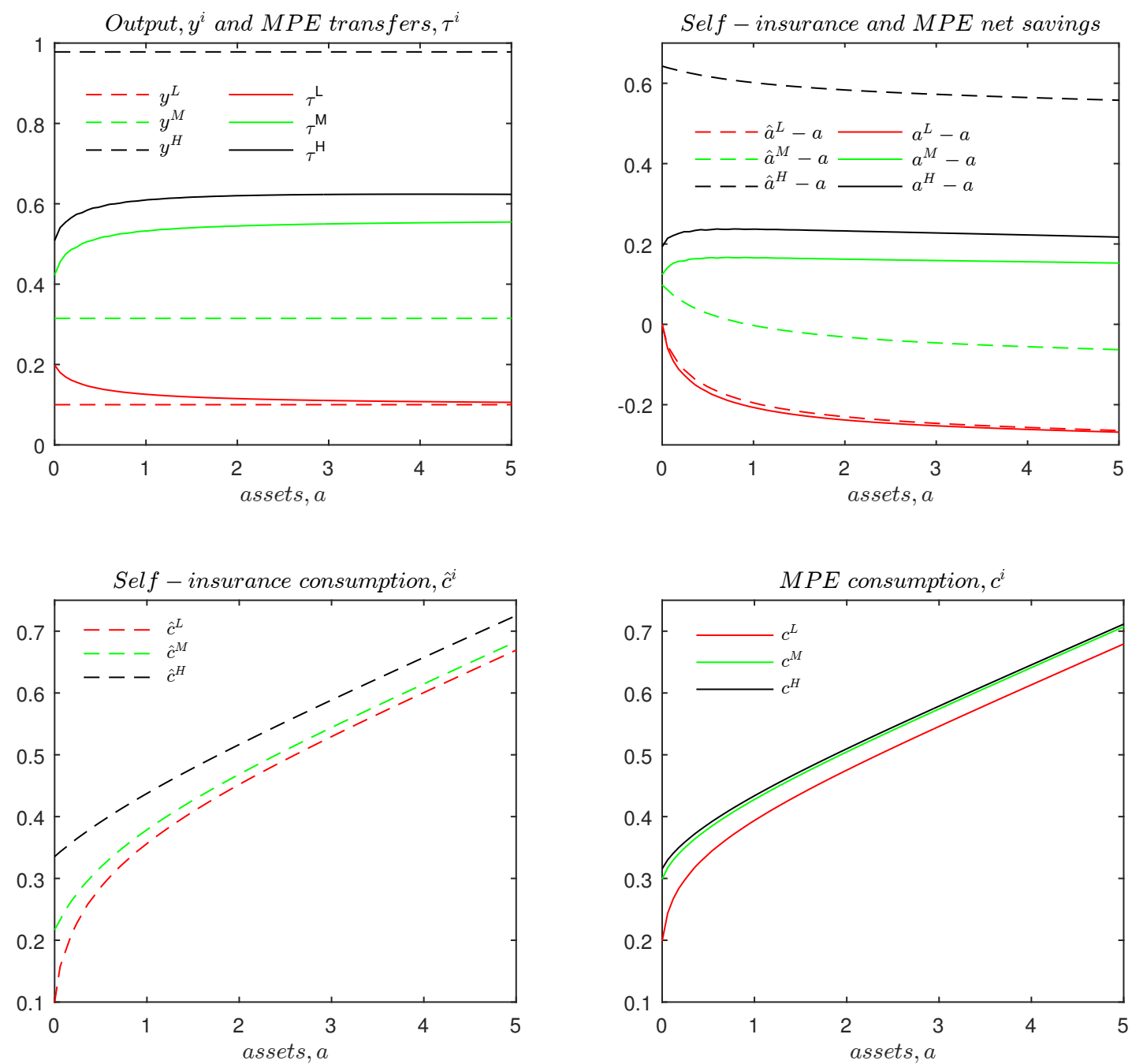

the insurer chooses instead to optimally use transfers to smooth consumption across states of the world. Indeed, the bottom two panels of Figure 1 show that the consumption levels in all three output states are much closer to each other in the MPE setting compared to those in self-insurance. Naturally, these consumption differences are most pronounced for low asset levels for which agents are most severely constrained in terms of their ability to smooth consumption on their own. ${ }^{17}$

Figure 2 compares effort, $e$ and expected non-asset income, defined as $\bar{y}(e) \equiv \sum_{i=1}^{n} \pi^{i}(e) y^{i}$ in the self-insurance setting and as $\bar{\tau}(e) \equiv \sum_{i=1}^{n} \pi^{i}(e) \tau^{i}$ in the MPE. In the self-insurance setting, effort and labor income are monotonically decreasing, due to the decreased demand for self-insurance by richer agents. In contrast, effort in the MPE economy varies non-monotonically with agent's assets

\footnotetext{
${ }^{17}$ Given the additional insurance in MPE compared to self-insurance, the agent's MPE effort $\mathcal{E}(a)$ in our simulation is strictly lower than in self-insurance and decreases in the agent's assets a (not depicted in the Figure). This is another mechanism which reduces MPE asset holdings since, for any given $a$, the probability of high output is lower in MPE compared to in self-insurance.
} 
Figure 2: Effort and Expected Non-Asset Income
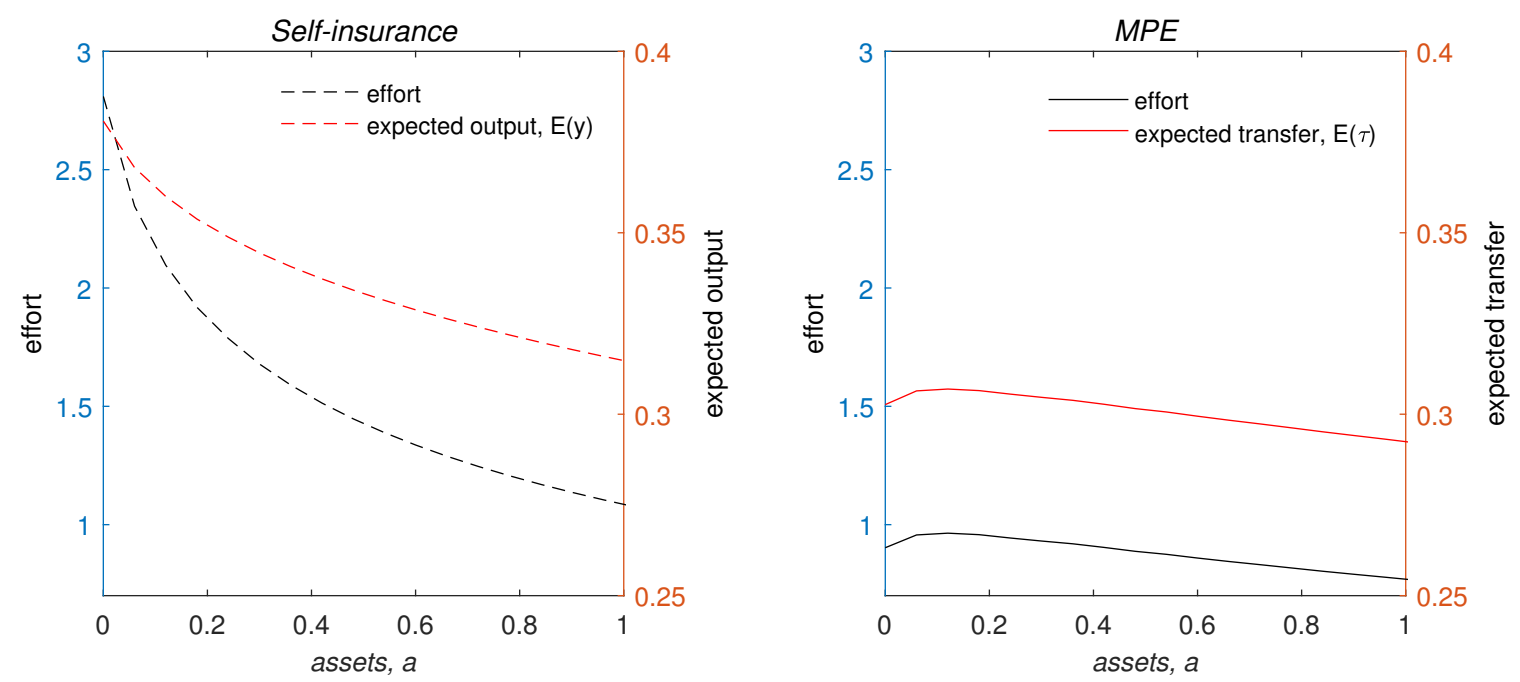

a. In particular, effort $e$ and expected "labor income", i.e., expected transfers, $\bar{\tau}(e)$ increase in the agent's assets for low asset levels, which in our calibration corresponds to the agents in the bottom third of the stationary asset distribution. This result shows that, unlike self-insurance, MPE insurance can generate a positive correlation between agent's assets and work effort, at least for a range of asset values. The reason for this result is the interaction between the incentivesinsurance trade-off and the borrowing constraint. ${ }^{18}$ We verified that the non-monotonicity is present regardless of how risk-averse the agent is, though naturally, the effect is more pronounced at higher levels of risk-aversion. The result does not obtain with full information (no incentive problem) or with risk neutrality (no demand for insurance), but does obtain under one-sided commitment. Finally, we note from Figure 2 that MPE insurance substantially flattens the profile of effort as function of wealth compared to self-insurance.

Figure 3 shows the aggregate degree of consumption smoothing using a panel of simulated data. Specifically, we generate data for 1,000 agents over 10 periods from each of the MPE and selfinsurance models, initialized at their respective long-run stationary asset distributions. Figure 3 plots the deviations from each period's average for each of the 1,000 agents for output, $y^{i}$ and for income and consumption, as defined in (3) and (4)-(5), respectively. In the MPE there is significant additional consumption smoothing compared to self-insurance which is achieved via the two distinct and complementary mechanisms outlined above. First, the comparison between the left and middle panels of Figure 3 shows that income in the MPE is significantly smoother than income in selfinsurance. Intuitively, the transfers $\mathcal{T}^{i}(a)$ (which are the main component of MPE income for low assets $a$ ) are optimally adjusted depending on the output history, unlike the exogenous component $y^{i}$ in self-insurance. Second, comparing the middle and right panels, we see that, out of the already smoother income in MPE, consumption is smoothed even further relative to self-insurance.

Figure 4 describes the dynamics of the main variables in the MPE and self-insurance economies. For each of the two settings, we plot the time-paths of simulated output, income, consumption and next-period assets of an agent with the same initial asset level, $a_{0}$ (set equal to the MPE long-

\footnotetext{
${ }^{18}$ If we decrease the lower bound on assets then the region of non-monotonicity shifts to the left.
} 
Figure 3: Income and Consumption Smoothing
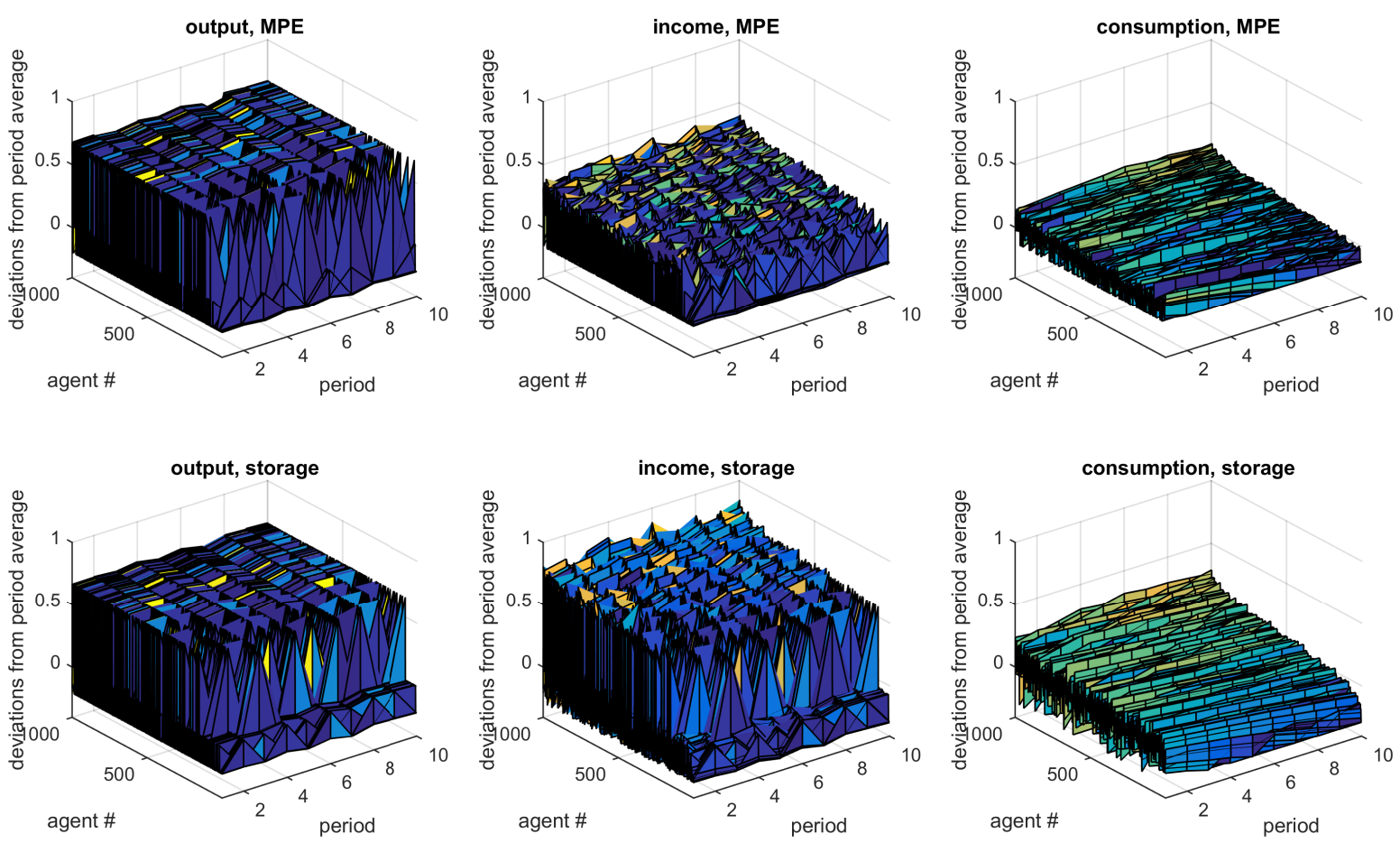

run median assets) and experiencing the exact same sequence of output realizations (the dotted line). In the upper row of panels labeled Example 1, the simulated output sequence in both settings is: medium output $\left(y^{M}\right)$ for three periods; low output $\left(y^{L}\right)$ for three periods; and medium output $\left(y^{M}\right)$ again for the last 4 periods, or $\{\mathrm{M}, \mathrm{M}, \mathrm{M}, \mathrm{L}, \mathrm{L}, \mathrm{L}, \mathrm{M}, \mathrm{M}, \mathrm{M}, \mathrm{M}\}$. We observe higher and smoother consumption in MPE, which is achieved by faster asset accumulation in "better times" (medium output) and running down assets in "bad times" (low output), in addition to appropriately varying the insurance transfers. In Example 2, the bottom row of panels in Figure 4, the common output sequence is instead $\{\mathrm{L}, \mathrm{H}, \mathrm{L}, \mathrm{M}, \mathrm{L}, \mathrm{M}, \mathrm{L}, \mathrm{H}, \mathrm{L}, \mathrm{M}\}$. Despite the very high variability of output, consumption is smoothed almost perfectly in Markov-perfect equilibrium but varies much more over time in the self-insurance economy. In Example 2 most of the smoothing in the MPE is done via the transfers.

\subsubsection{Long-run properties}

The MPE and self-insurance settings yield significantly different long-run wealth distributions. The Gini coefficient of the wealth (assets) distribution is 0.45 in the MPE compared to 0.35 in the selfinsurance economy. As a comparison, Aiyagari's (1994) canonical self-insurance model with an exogenous persistent output process yields a Gini coefficient of 0.38 while the wealth Gini in US data is estimated at 0.80 (Castañeda et al., 2003).

Investigating the wealth distribution implications further, in Figure 5 we display the Lorenz curves in the MPE and the self-insurance economies. Most of the difference and the higher level of inequality in the MPE are explained by the fact that low-wealth agents have weaker incentives to 
Figure 4: Time Paths
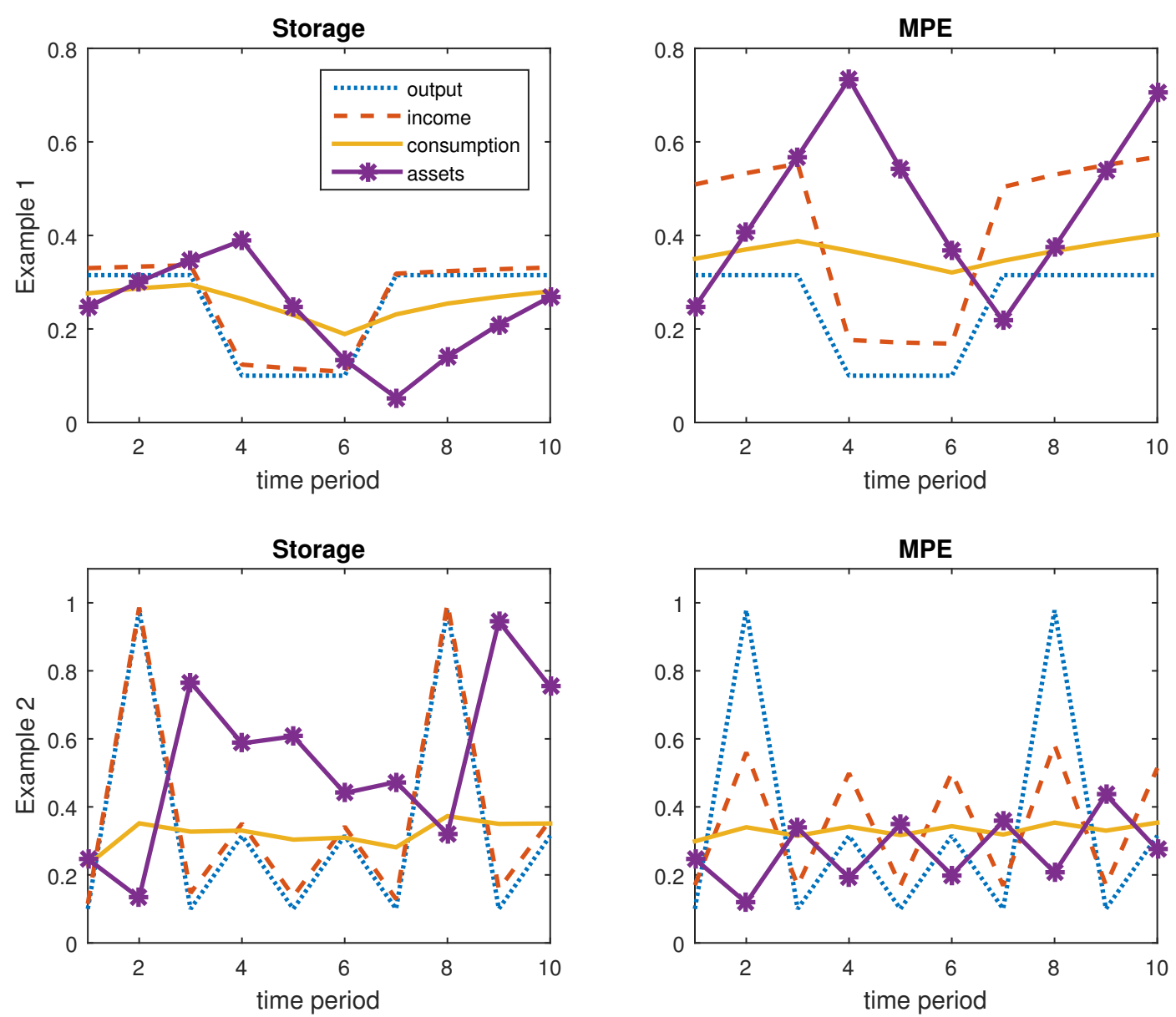

self-insure through asset accumulation. Indeed, the median agent in the long-run assets distribution is significantly less wealthy in the Markov-perfect setting compared to his counterpart in the selfinsurance economy (asset holdings of 0.25 vs. 1.12, respectively). ${ }^{19}$ Intuitively, the additional insurance in the MPE decreases the demand for buffer-stock savings. In contrast, in the selfinsurance economy the risk-averse agents work hard to avoid ending up with zero or very low assets since they would be unable to smooth consumption after a low-output realization.

Figure 6 plots the histogram of the long-run stationary asset distribution in the MPE. The distribution replicates well the long right tail and significant mass of agents near zero wealth in US data (see, for example, Chart 3 in Budría-Rodríguez et al., 2002). The reason for the large mass of agents near zero assets in the MPE is the consumption insurance provided via transfers.

Finally, Figure 7 compares the long-run stationary distributions of consumption in the MPE and self-insurance economies. We find that the long-run consumption histogram with Markov-perfect insurance is qualitatively very similar to the consumption histogram in the private-information limited-enforcement ("PILE") model of Broer et al. (2017) - see Figure 3 in their paper. Furthermore, the skewness of log consumption has the same sign and similar magnitude: -0.77 in our

\footnotetext{
${ }^{19}$ The median-assets agent in MPE has asset holdings equal to $80 \%$ of his mean income compared to the medianassets agent in self-insurance who holds assets three times larger than his mean income.
} 
Figure 5: Lorenz Curves

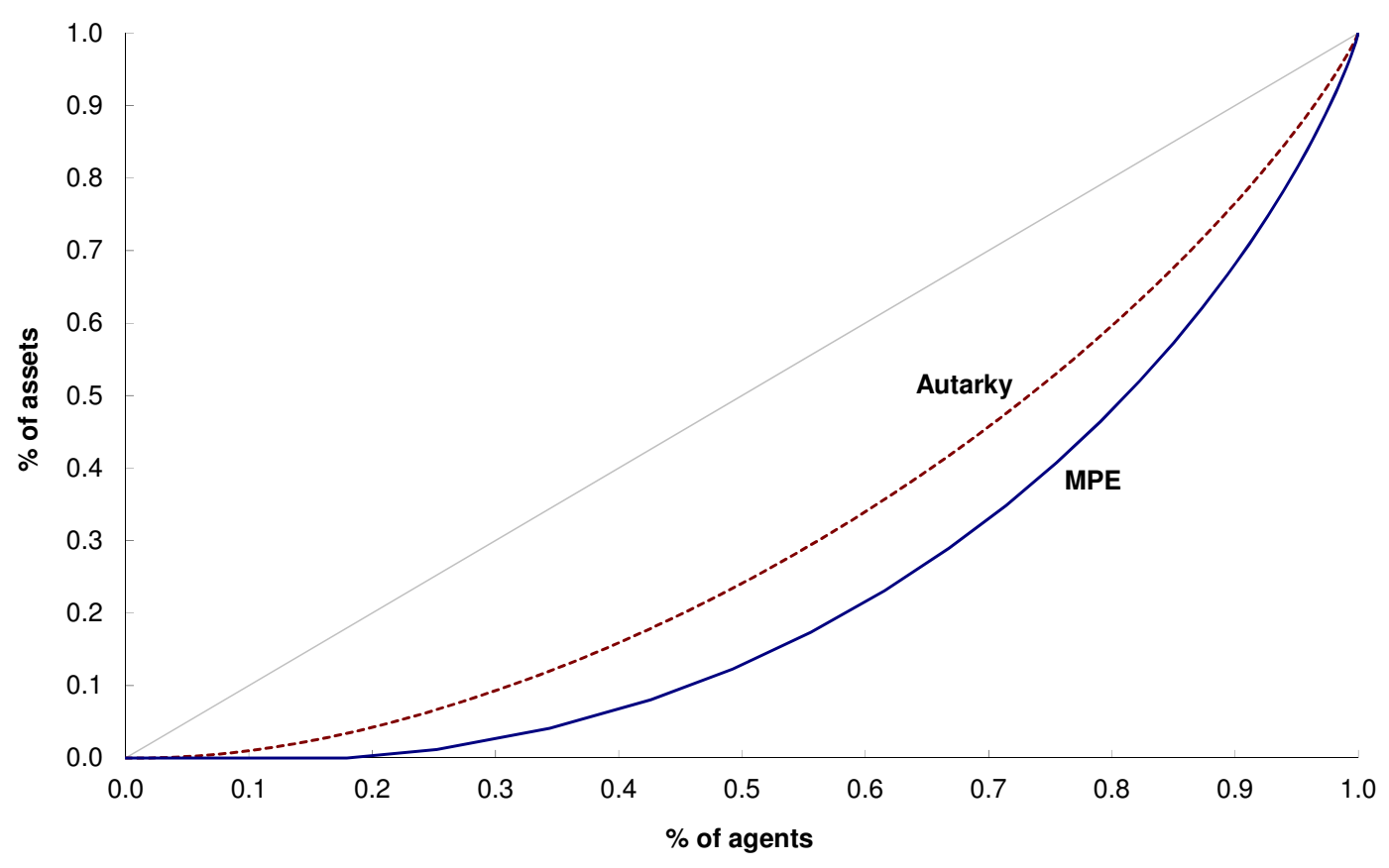

Figure 6: Asset Histogram

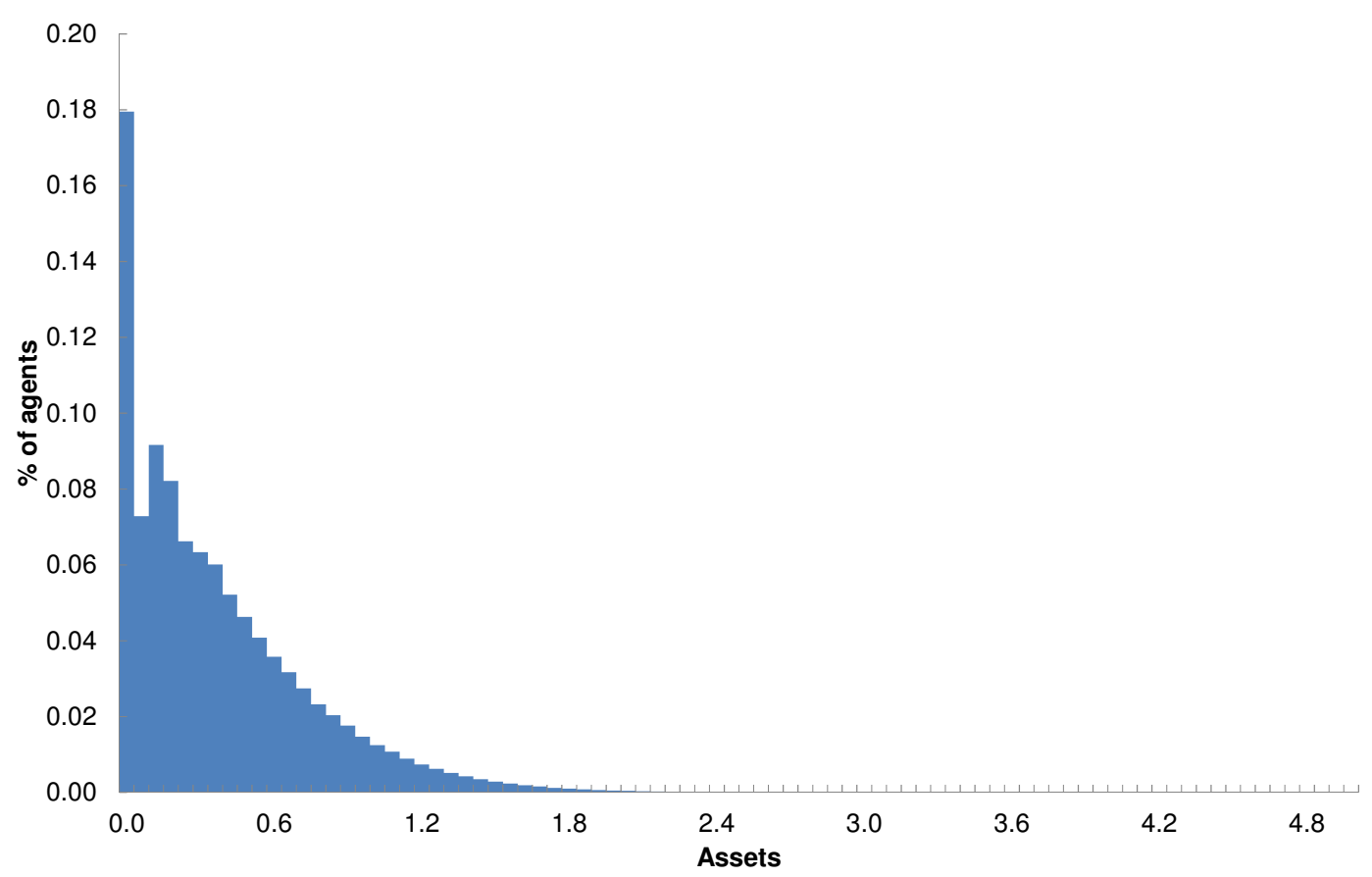


Figure 7: Consumption Histogram
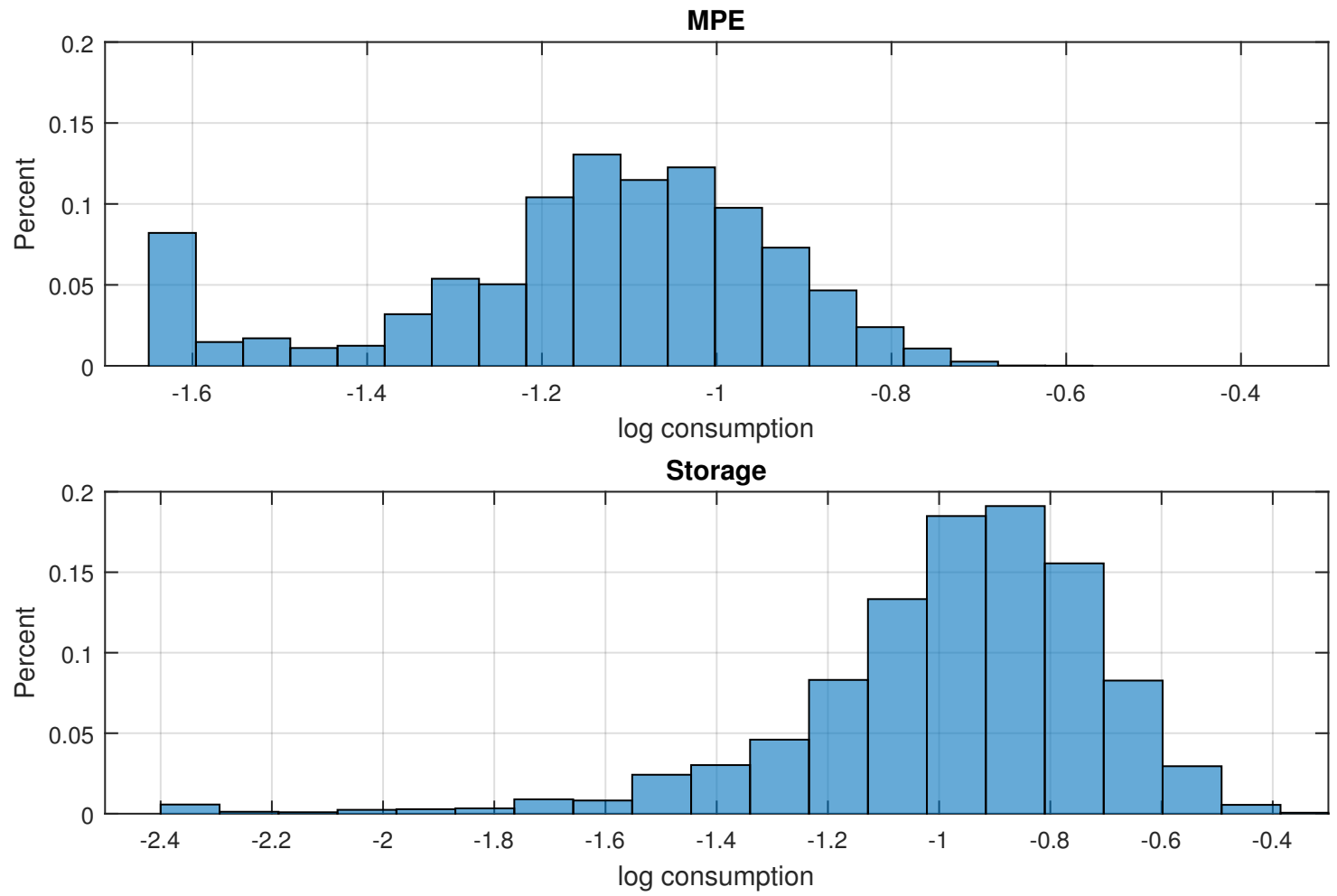

Markov-perfect insurance setting vs. -0.94 in Broer et al. (2017). These similarities obtain despite the fact that Broer et al. (2017) model unobserved income as opposed to unobserved action, a different limited enforcement friction, a different equilibrium/solution concept and a different persistent income process (exogenous Markov chain). In addition, the long-run consumption distribution in our self-insurance economy is much more left-skewed (the skewness of $\ln c$ equals -1.50 ), much as in the self-insurance with natural borrowing limit model ("SIN") in Broer et al. (2017) with reported skewness of $\ln c$ equal to -1.67 or -1.82 , depending on the specification. We cautiously interpret these results as further validation of our Markov-perfect insurance model in relation to the recent literature.

\section{$5 \quad$ Welfare analysis}

Our Markov-perfect dynamic risk-sharing setting features two important frictions: private information and limited commitment. In this section, we evaluate and compare the efficiency losses associated with each of these two frictions for different asset levels in our parameterization. Specifically, for all $a \in \mathbb{A}$ we solve for the one-time consumption equivalent compensation function $\Delta(a)$ defined as

$$
\sum_{i=1}^{n} \pi^{i}(\mathcal{E}(a))\left\{u\left(\mathcal{C}^{i}(a)[1+\Delta(a)]\right)+\beta \Omega\left(\mathcal{A}^{i}(a)\right)\right\}-\mathcal{E}(a)=\tilde{\mathcal{V}}(a)
$$


where $\left\{\mathcal{C}^{i}(a), \mathcal{A}^{i}(a), \mathcal{E}(a), \mathcal{V}(a)\right\}_{i=1}^{n}$ correspond to the MPE from Definition 1 and $\tilde{\mathcal{V}}(a)$ is the agent's value in an alternative insurance regime obtained by removing one of the frictions, that is, either (a) a setting with full information (no moral hazard) and limited commitment or (b) a setting with private information and full commitment. We interpret $\Delta(a)$ as the one-time welfare gain in terms of current consumption associated with resolving either the information or commitment frictions, relative to our baseline MPE with both frictions.

We first compute the welfare gains from resolving the information (moral hazard) friction. A Markov-perfect equilibrium with full information, that is, when agent's effort is observable and contractible, is defined in a way analogous to Definition 1 but without the incentive compatibility constraint (see Section 3.3). Second, we compute the welfare gains from resolving the commitment friction by solving the dynamic risk-sharing problem with full (two-sided) commitment, that is, assuming both the insurer and the agent can commit to a lifetime agreement at time zero. ${ }^{20}$ This full-commitment problem is formulated like the one-sided commitment problem in Section 3.4 and Appendix 7.2, but allowing promised utility values smaller than $\mathcal{V}(0)$ (possibly down to $-\infty$ ). We compute the equivalent compensation function $\Delta(a)$ defined in (6) for each of the two scenarios. This means replacing $\tilde{\mathcal{V}}(a)$ with the corresponding value function from either the full-information (with limited commitment) or full-commitment (with moral hazard) solutions.

Figure 8 displays the welfare gains from resolving the information and commitment frictions, plotted against the agent's asset level, $a$. The welfare gains from resolving the private information friction are decreasing in $a$ but remain significant at all asset levels. Going to the setting with full information and limited commitment at $a=0$ would result in a welfare gain equivalent to a onetime consumption increase of over $200 \%$, holding all else equal. Intuitively, with full information the moral hazard problem is absent and the agent receives full instead of partial insurance, which is especially valued at low asset levels. In our parameterization, the value of additional insurance measured by the gains from resolving the moral hazard problem is still significant at high asset levels (e.g., it is about $15 \%$ at $a=5$ ).

Endowing both the insurer and the agent with commitment power also results in significant welfare gains (see Figure 8). Recall from Section 3.4 that endowing only the insurer with longterm commitment does not affect the resulting allocations (Proposition 3). Therefore, what is key for the efficiency gains from commitment is the inability of the agent to walk away from the risk-sharing contract. This allows the insurer to front-load the agent's consumption and provide better incentives to supply effort. The estimated welfare gains from two-sided commitment to a long-term insurance contract are large, especially at low assets: at $a=0$ a move from Markovperfect insurance to the full commitment setting results in welfare gain equivalent to a one-time consumption increase of over $300 \%$. The gains from commitment decrease rapidly as the agent's asset holdings increase and eventually converge to zero.

Figure 8 also compares head-to-head the welfare gains from resolving the information and commitment frictions. For our parameterization to US data the gains from resolving the commitment friction are larger than the gains from resolving the moral hazard friction at low asset levels. The opposite holds at high asset levels. The intuition is as follows. In the presence of moral hazard,

\footnotetext{
${ }^{20}$ Recall that insurance with one-sided commitment by the insurer is equivalent in terms of allocations to Markovperfect insurance - see Proposition 3. We verify this result numerically by comparing the value function of the MPE with the first-stage one-sided commitment value function, for all asset levels: the maximum numerical error between value functions is equivalent to a one-time consumption increase of only $0.04 \%$, which occurs at assets levels very close to zero, where the lower bounds on savings and promised utility bind; the error is essentially zero elsewhere. Given this result, we assess the welfare gains of resolving both the insurer's and the agent's commitment problems simultaneously.
} 
Figure 8: Welfare Gains from Resolving the Information or Commitment Frictions

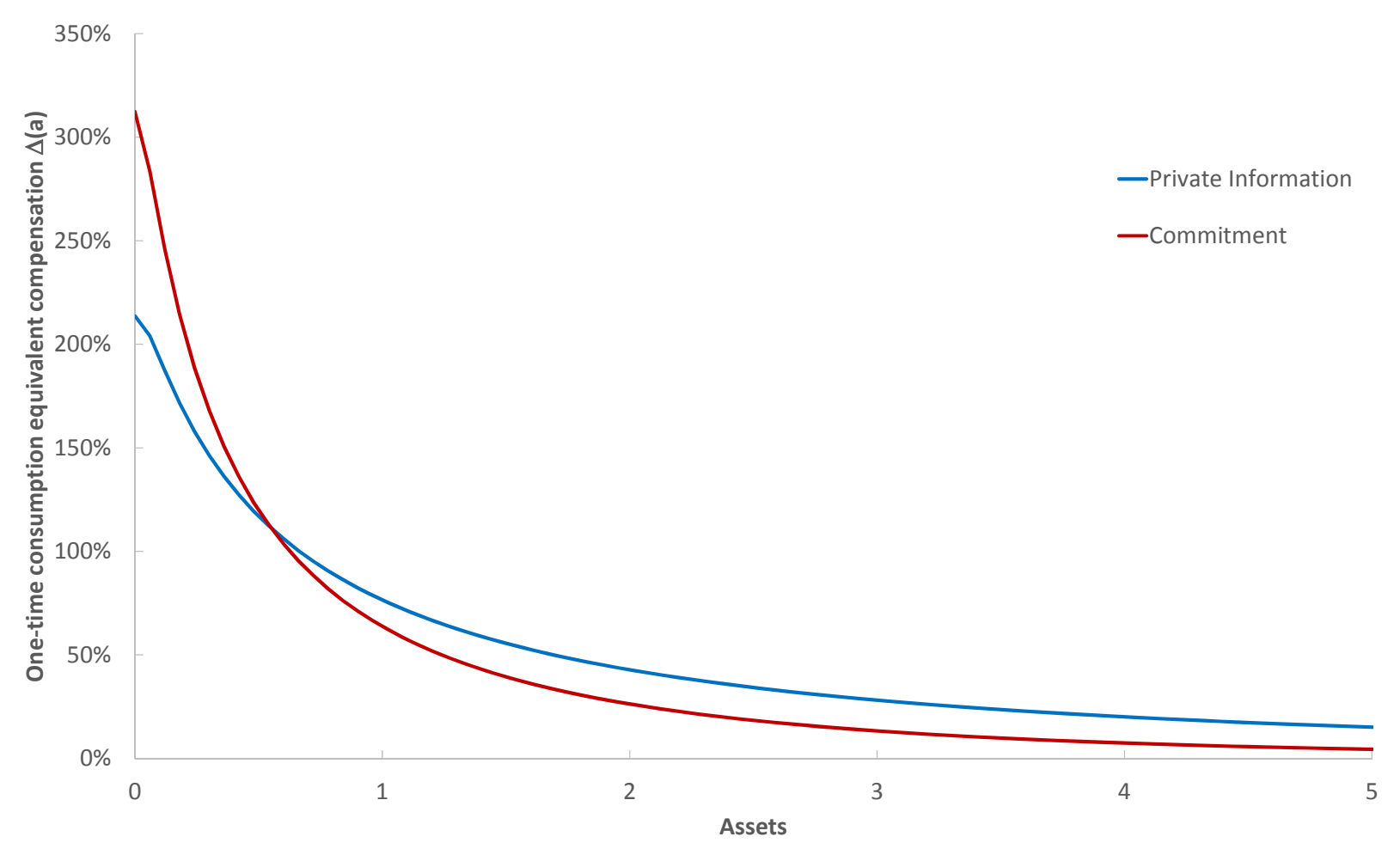

Note: $\Delta(a)$ solves (6) for each case.

the gains from commitment are associated with the insurer's ability to immiserate the agent in the long-run by front-loading consumption. Hence, these gains depend crucially on the agent's demand for outside insurance which is function of the agent's assets. The demand for insurance is stronger for low assets and hence the gains from commitment are larger. In contrast, the welfare gains from resolving the moral hazard friction arise from making effort contractible. This is particularly beneficial at larger asset levels, for which it is harder to induce high effort by the agent.

\section{Conclusions}

Understanding how individuals cope with idiosyncratic risk is a fundamental economic problem with policy-relevant implications. Savings are a natural vehicle for insurance against income fluctuations and expenditure shocks. However, self-insurance via asset accumulation generally does not allow agents to hedge against all contingencies. The resulting demand for additional risk sharing is covered by private contracts or public insurance policies. We highlight two fundamental frictions which afflict the scope of insurance: private information and limited commitment. Furthermore, an individual's wealth affects the bite both these frictions have in the design of insurance contracts.

We propose Markov-perfect equilibria as a solution concept to characterize dynamic risk-sharing contracts subject to private information and limited commitment. We show that Markov-perfect 
insurance contracts preserve standard properties of optimal contracts with private information and are not more restrictive than long-term insurance contracts with one-sided commitment by the insurer. An important advantage of our approach is that the terms of Markov-perfect insurance contracts (insurance premia or payments) are expressed entirely in terms of observable and measurable variables: output (income) and the insured agent's assets. This stands in contrast to many papers on dynamic contracts which rely on unobservable state variables ("promised utility") and, importantly, opens up new venues for quantitative and empirical analysis, some of which we explore here. In particular, endogenous asset accumulation is an integral part of Markov-perfect insurance contracts and hence, our model delivers implications for the dynamics and distribution of wealth in an economy with endogenously incomplete markets, in addition to the implications for consumption and income smoothing emphasized in the existing private information and limited commitment literatures. $^{21}$

While we have not yet investigated the full quantitative implications of Markov-perfect risksharing contracts beyond the presented examples, we believe that our approach offers a viable and tractable computational framework for dynamic macroeconomic analysis of risk sharing subject to commitment and information constraints. Elements such as a persistent income process with permanent and transitory shocks or various public policy schemes (e.g., taxes, social security, etc.) can be added to the basic setting while preserving its relatively low computational burden.

\footnotetext{
${ }^{21}$ The computational tractability of Markov-perfect insurance is well-suited for potential structural estimation work using consumption, income and wealth data, for example following Karaivanov and Townsend (2014).
} 


\section{References}

[1] Abraham, A. and N. Pavoni (2008), "Efficient Allocations with Moral Hazard and Hidden Borrowing and Lending: A Recursive Formulation", Review of Economic Dynamics 11(4): 781-803.

[2] Aiyagari, S.R. (1994), "Uninsured Idiosyncratic Risk and Aggregate Saving", Quarterly Journal of Economics 109(3): 659-84.

[3] Albanesi, S. and C. Sleet (2006), "Dynamic Optimal Taxation with Private Information", Review of Economic Studies 73(1): 1-30.

[4] Allen, F. (1985), "Repeated principal-agent relationships with lending and borrowing", Economics Letters 17(1-2): 27-31.

[5] Atkeson, A. and R. Lucas (1992), "On efficient distribution with private information", Review of Economic Studies 59(3): 427-53

[6] Bolton, P. and M. Dewatripont (2004), Contract Theory, The MIT Press, Cambridge, MA.

[7] Broer, T., M. Kapicka and P. Klein (2017), "Consumption risk sharing with private information and limited enforcement", Review of Economic Dynamics 23: 170-90.

[8] Budría-Rodríguez, S., J. Díaz-Gimémez, V. Quadrini and J.-V. Ríos-Rull (2002), "Updated Facts on the U.S. Distributions of Earnings, Income, and Wealth", Federal Reserve Bank of Minneapolis Quarterly Review 26(3): 2-35.

[9] Castañeda, A., J. Díaz-Gimémez and J.-V. Ríos-Rull (2003), "Accounting for the U.S. Earnings and Wealth Inequality", Journal of Political Economy 111(4): 818-57.

[10] Cole, H. and N. Kocherlakota (2001), "Efficient Allocations with Hidden Income and Hidden Storage", Review of Economic Studies 68(3): 523-42.

[11] Fudenberg, D., B. Holmstrom and P. Milgrom (1990), "Short-Term Contracts and Long-Term Agency Relationships", Journal of Economic Theory 51(1): 1-31.

[12] Golosov, M., A. Tsyvinski and I. Werning (2006), "New Dynamic Public Finance: A User's Guide", NBER Macroeconomics Annual, vol. 21: 317-88, National Bureau of Economic Research.

[13] Green, E. (1987), "Lending and Smoothing of Uninsurable Income", in E.C. Prescott and N. Wallace (eds), Contractual Arrangements for Intertemporal Trade pp. 3-25. University of Minnesota Press.

[14] Karaivanov, A. and F. Martin (2015), "Dynamic Optimal Insurance and Lack of Commitment", Review of Economic Dynamics 18(2): 287-305.

[15] Karaivanov, A. and F. Martin (2016), "Market Power and Asset Contractibility in Dynamic Insurance Contracts", Federal Reserve Bank of St. Louis Review 98(2): 111-27.

[16] Karaivanov, A. and R. Townsend (2014), "Dynamic Financial Constraints: Distinguishing Mechanism Design from Exogenously Incomplete Regimes", Econometrica 82(3): 887-959. 
[17] Khan, A., L. Popov and B. Ravikumar (2014), "Enduring Relationships in an Economy with Capital and Private Information", working paper.

[18] Kocherlakota, N. (1996), "Implications of Efficient Risk Sharing without Commitment", Review of Economic Studies 63(4): 595-609.

[19] Krueger, D. and H. Uhlig (2006), "Competitive risk-sharing contracts with one-sided commitment", Journal of Monetary Economics 53(7): 1661-91.

[20] Ligon, E., J. Thomas and T. Worrall, (2002), "Informal Insurance Arrangements with Limited Commitment: Theory and Evidence from Village Economies", Review of Economic Studies 69(1): 209-44.

[21] Ljungqvist, L. and T. Sargent (2004), Recursive Macroeconomic Theory, 2nd edition, MIT Press.

[22] Malcomson, J. and F. Spinnewyn (1988), "The Multiperiod Principal-Agent Problem", Review of Economic Studies 45: 391-408.

[23] Maskin, E. and J. Tirole (2001), "Markov Perfect Equilibrium", Journal of Economic Theory 100(2): 191-219.

[24] Meh, C. and V. Quadrini (2006), "Endogenous market incompleteness with investment risks", Journal of Economic Dynamics and Control 30(11): 2143-65.

[25] Phelan, C. (1995), "Repeated Moral Hazard and One-Sided Commitment", Journal of Economic Theory 66(2): 488-506.

[26] Phelan, C. (1998), "On the Long Run Implications of Repeated Moral Hazard", Journal of Economic Theory 79(2): 174-91.

[27] Phelan, C. and R. Townsend (1991), "Computing Multi-Period Information-Constrained Equilibria", Review of Economic Studies 58: 853-81.

[28] Rogerson, W. (1985a), "The First Order Approach to Principal-Agent Problems", Econometrica 53(1): 1357-68.

[29] Rogerson, W. (1985b) "Repeated Moral Hazard", Econometrica 53: 69-76.

[30] Spear, S. and S. Srivastava (1987), "On Repeated Moral Hazard with Discounting", Review of Economic Studies 54(4): 599-617.

[31] Thomas, J. and T. Worrall (1988), "Self-Enforcing Wage Contracts", Review of Economic Studies 55(4): 541-53.

[32] Thomas, J. and T. Worrall (1990), "Income fluctuation and asymmetric information: An example of a repeated principal-agent problem", Journal of Economic Theory 51(2): 367-90.

[33] Thomas, J. and T. Worrall (1994), "Foreign Direct Investment and the Risk of Expropriation", Review of Economic Studies 61(1): 81-108.

[34] Townsend, R. (1982), "Optimal Multiperiod Contracts and the Gain from Enduring Relationships Under Private Information", Journal of Political Economy 90(6): 1166-86. 


\section{Appendix}

\subsection{Proofs of Propositions 1 and 2}

Proof of Proposition 1. With Lagrange multipliers $\lambda$ and $\mu$ on (IC) and (ZP) respectively, the first-order conditions with respect to transfers and assets are

$$
\begin{aligned}
u_{c}\left(c^{i}\right)\left[\pi^{i}(e)+\lambda \pi_{e}^{i}(e)\right]-\mu \pi^{i}(e) & =0 \\
{\left[-u_{c}\left(c^{i}\right)+\beta \mathcal{V}_{a}\left(a^{i}\right)\right]\left[\pi^{i}(e)+\lambda \pi_{e}^{i}(e)\right] } & =0
\end{aligned}
$$

for all $i=1, \ldots, n$. Since, by Assumption $1, \pi^{i}(e)$ are such that the first-order approach is valid, the incentive constraint (IC) binds at optimum and $\lambda>0$ - see Rogerson (1985a) or Bolton and Dewatripont (2004) for discussion and proofs.

We also show that $\mu>0$. Indeed, re-arrange (7) as,

$$
\frac{\mu \pi^{i}(e)}{u_{c}\left(c^{i}\right)}=\pi^{i}(e)+\lambda \pi_{e}^{i}(e)
$$

and sum over $i=1, \ldots, n$ to obtain

$$
\mu \sum_{i=1}^{n} \frac{\pi^{i}(e)}{u_{c}\left(c^{i}\right)}=\sum_{i=1}^{n} \pi^{i}(e)+\lambda \sum_{i=1}^{n} \pi_{e}^{i}(e) .
$$

Given that $\sum_{i=1}^{n} \pi^{i}(e)=1$ for all $e$, we have $\sum_{i=1}^{n} \pi_{e}^{i}(e)=0$, which implies

$$
\mu=\frac{1}{E\left[\frac{1}{u_{c}(c)}\right]}>0 \text {. }
$$

Next, re-arrange (7) as

$$
\frac{\mu}{u_{c}\left(c^{i}\right)}=1+\lambda \frac{\pi_{e}^{i}(e)}{\pi^{i}(e)}
$$

which, given Assumption 1 (MLRP) and given $\mu, \lambda>0$ and the strict concavity of $u(c)$, implies $c^{1} \leq c^{2} \leq \ldots \leq c^{n}$. Since this is true for any $a \in \mathbb{A}$, the result in part (i) follows.

(ii) The second result, the inequalities between $\mathcal{C}^{i}(a)$ and $\mathcal{C}^{j}(a)$, follows directly from (10) using that $\mu, \lambda>0$ and the strict concavity of $u(c)$. We prove the first result, partial insurance, by contradiction. We know, by part (i) that $\mathcal{C}^{1}(a) \leq \mathcal{C}^{n}(a)$. Suppose $c^{1}=\mathcal{C}^{1}(a)=\mathcal{C}^{n}(a)=c^{n}$. Then, from $(10)$, it must be that $\frac{\pi_{e}^{i}(e)}{\pi^{i}(e)}$ are equal for all $i=1, \ldots, n$, that is,

$$
\pi_{e}^{i}(e)=m \pi^{i}(e)
$$

for some constant $m$. Suppose $m \neq 0$. But then, summing the above equalities over $i$, we obtain a contradiction since the LHS adds up to 0 while the RHS adds up to $m$ for any $e$. The case $m=0$ is ruled out by Assumption 1(ii and iv). Therefore, $\mathcal{C}^{1}(a)<\mathcal{C}^{n}(a)$ and there is partial insurance in MPE.

(iii) and (iv) Note first that, given $u_{c}\left(c^{i}\right)>0$ and $\mu>0,(7)$ implies $\pi^{i}(e)+\lambda \pi_{e}^{i}(e)>0$ for all $i=1, \ldots, n$. Thus, using (8) we obtain:

$$
-u_{c}\left(c^{i}\right)+\beta \mathcal{V}_{a}\left(a^{i}\right)=0 \quad \forall i
$$


Observe that (11) is equivalent to the first-order condition with respect to future assets, $a^{i}$ in the agent's objective function. That is, for any $a$, the MPE implies future asset level, $a^{i}=\mathcal{A}^{i}(a)$ that is the same as the asset level which the agent would choose if assets were non-contractible.

The envelope condition implies

$$
\mathcal{V}_{a}(a)=r \sum_{i=1}^{n} u_{c}\left(c^{i}\right)\left[\pi^{i}(e)+\lambda \pi_{e}^{i}\right],
$$

which, using (7), yields $\mathcal{V}_{a}(a)=r \mu$. Replace $\mu$ from (9) and update one period ahead. Substituting the resulting expression into (11) yields the inverse Euler equations.

Finally, using $\mathcal{V}_{a}(a)=r \mu$ and $\mu>0$, the value function $\mathcal{V}(a)$ is strictly increasing on $i n t \mathbb{A}$. We use this result in Proposition 3.

Proof of Proposition 2. (i) Taking the first order conditions with respect to $\tau^{i}$ immediately yields $c^{i}=c^{j}=c$ for all $i, j=1, . . n$. Thus, using (ZP), we obtain $\tau^{i}=\bar{y}(e)$ for all $i=1 \ldots, n$. The FOCs with respect to $a^{i}$ are symmetric across the output states. Therefore, assuming a symmetric solution (existence is ensured as discussed in the main text), $a^{i}=a^{j}=a^{\prime}$ for all $i, j=1, . . n$.

Given the above, solving the Markov-perfect insurance problem with full information is equivalent to solving the following dynamic programming problem:

$$
\bar{V}(a)=\max _{a^{\prime}, e} u\left(r a+\bar{y}(e)-a^{\prime}\right)+\beta \bar{V}\left(a^{\prime}\right)-e
$$

where $\bar{y}(e) \equiv \sum \pi^{i}(e) y^{i}$ denotes expected output at effort level $e$.

(ii) The Euler equation is derived in the standard way from the FOC with respect to $a^{\prime}$ at an interior solution:

$$
-u_{c}(c)+\beta \bar{V}_{a}\left(a^{\prime}\right)=0
$$

and the envelope condition: $\bar{V}_{a}(a)=r u_{c}(c)$.

(iii-v) Since $\beta r<1$ and $u(c)$ is strictly concave, the Euler equation in (ii) implies $c^{\prime}<c$, or equivalently, $\mathcal{C}(\mathcal{A}(a))<\mathcal{C}(a)$. To show the remaining results, we use the following auxiliary lemma.

Lemma S1: Expected output $\bar{y}(e)$ is strictly increasing and concave in e.

Proof. Expected output is strictly increasing since, using Assumption 1, it is well-known that MLRP implies first order stochastic dominance; thus $e_{1}>e_{2}$ implies $\bar{y}\left(e_{1}\right)>\bar{y}\left(e_{2}\right)$ and hence $\bar{y}(e)$ is strictly increasing. By the definition of $\pi^{i}(e)$, differentiating $\sum_{i=1}^{n} \pi^{i}(e)=1$ twice with respect to $e$, we obtain $\sum_{j=1}^{n} \pi_{e e}^{i}(e)=0$ for all $e \in E$ and hence, using Assumption 1(vi), the partial sums from $j$ to $n$ satisfy $\sum_{j}^{n} \pi_{e e}^{j}(e) \leq 0$ for $j=2, \ldots, n$ and all $e \in \mathbb{E}$. To show that $\bar{y}(e)$ is concave, compute the derivative:

$$
\begin{aligned}
\frac{d^{2} \bar{y}(e)}{d e^{2}} & =\sum_{i=1}^{n} \pi_{e e}^{i}(e) y^{i}= \\
& =\sum_{j=1}^{n} \pi_{e e}^{j}(e) y^{1}+\sum_{j=2}^{n} \pi_{e e}^{j}(e)\left(y^{2}-y^{1}\right)+\ldots+\pi_{e e}^{n}(e)\left(y^{n}-y^{n-1}\right) \leq 0
\end{aligned}
$$

The derivative is non-positive since the first term in the sum is zero while all other terms are $\leq 0$ by the non-positivity of the partial sums shown above. 
Re-write problem (13) as:

$$
\begin{aligned}
\bar{V}(a) & =\max _{c, e, a^{\prime}} f(c, e)+\beta \bar{V}\left(a^{\prime}\right) \\
\text { s.t. } a^{\prime} & \leq g(a, c, e)
\end{aligned}
$$

where $f(c, e)=u(c)-e$ is the return function and $g(a, c, e)=r a+\bar{y}(e)-c$ is the feasibility constraint. Since $u(c)$ is strictly concave, $f(c, e)$ is strictly concave. In addition, the set $\left\{\left(a, a^{\prime}\right)\right.$ such that $\left.a^{\prime} \leq g(a, c, e)\right\}$ for all admissible $(c, e)$ is convex and compact since $\mathbb{A}$ is compact and $a$ enters $g$ linearly. By standard arguments (e.g., Ljungqvist and Sargent, 2004), these conditions imply that the functional equation has a unique strictly concave solution $\bar{V}(a)$.

Now go back to the FOC with respect to $a^{\prime}$ in (13),

$$
u_{c}(c)=\beta \bar{V}_{a}\left(a^{\prime}\right)
$$

Since both $u$ and $\bar{V}$ are strictly concave, the FOC implies that $c$ and $a^{\prime}$ must change in the same direction as $a$ varies (both go up or both go down). The FOC with respect to effort $e$ is

$$
u_{c}(c) \frac{d \bar{y}(e)}{d e}=1
$$

which, by the concavity of $\bar{y}(e)$ shown in Lemma S1 and the strict concavity of $u(c)$, implies that $c$ and $e$ must change in opposite directions as $a$ varies. We use these results regarding how $c, e$ and $a^{\prime}$ change in $a$ to prove that $c$ and $a^{\prime}$ are strictly increasing in agent's assets $a$ while effort $e$ is decreasing in $a$. Suppose not, that is, suppose $c$ and $a^{\prime}$ both decrease in $a$ while $e$ increases in $a$ and consider the zero profit constraint,

$$
a^{\prime}+c=r a+\bar{y}(e) .
$$

If $c$ and $a^{\prime}$ were decreasing in $a$, the l.h.s. goes down in $a$ while the r.h.s. goes up, which would contradict the optimality of $c, a^{\prime}$ since we could increase $c$ and achieve a higher value for the objective - a contradiction. Therefore, the consumption and asset policy functions, $c=\mathcal{C}(a)$ and $a^{\prime}=\mathcal{A}(a)$ are strictly increasing in $a$ while effort, $e=\mathcal{E}(a)$ is decreasing in $a$.

Finally, the result $\mathcal{A}(a)<a$ follows from the previously shown results that $\mathcal{C}(a)$ is strictly increasing in $a$ and that $\mathcal{C}(\mathcal{A}(a))<\mathcal{C}(a)$.

\subsection{Proof of Proposition 3}

\subsubsection{Recursive formulation of risk sharing with commitment}

The contracts with full (two-sided) or one-sided commitment described in Section 3.4 can be written as a two-stage recursive problem in which the agent's assets are extracted by the insurer in the initial period (the agent's future asset holdings are set to $\underline{a}=0$ ) and 'replaced' by promises to deliver future utility via transfers. In the first stage, the insurer solves a static problem of offering the agent the maximum possible utility subject to zero ex-ante expected profits and incentive-compatibility.

As in Phelan (1995) or Krueger and Uhlig (2006), suppose the feasible values for promised utility in the commitment setting belong to the set $W=[\underline{w}, \bar{w}]$. The bounds of the set $W$ are, in 
general, endogenous. For the moment we treat $W$ as given and discuss it in more detail below. The first-stage $(t=0)$ problem is

$$
\mathcal{V}^{C}\left(a_{0}\right) \equiv \max _{e_{0},\left\{c_{0}^{i}, w_{1}^{i}\right\}_{i=1}^{n}} \sum_{i=1}^{n} \pi^{i}\left(e_{0}\right)\left[u\left(c_{0}^{i}\right)+\beta w_{1}^{i}\right]-e_{0}
$$

subject to

$$
\begin{array}{r}
\sum_{i=1}^{n} \pi_{e}^{i}\left(e_{0}\right)\left[u\left(c_{0}^{i}\right)+\beta w_{1}^{i}\right]-1=0 \\
\sum_{i=1}^{n} \pi^{i}\left(e_{0}\right)\left[y^{i}+r a_{0}-c_{0}^{i}+r^{-1} \Pi^{C}\left(w_{1}^{i}\right)\right]=0 .
\end{array}
$$

The insurer sets the agent's future asset holdings, $a_{1}^{i}=0$ and, in exchange, promises the agent state-dependent lifetime utility $w_{1}^{i}$ from period 1 onward. The first constraint ensures incentivecompatibility of the effort choice. The second constraint is the zero ex-ante profits condition implied by the free entry assumption. The insurer discounts future profits by $r^{-1}$ which can be interpreted as having the same savings technology as the agent.

With one-sided commitment, the agent can walk away at the beginning of each period. Thus, the promised utility $w_{1}^{i}$ with which the agent exits the first-stage problem must be at least as large as the agent's outside option, which we assume equals the agent's (endogenous) value of contracting with another insurer, carrying zero assets. ${ }^{22}$ Hence, in the one-sided commitment setting, the lower bound of the set of feasible promised utilities equals the value of the agent's outside option, $\underline{w}=v^{\text {out }}$ which we discuss in detail below. The following constraint then ensures the agent remains in the contract from $t=1$ on, for any realization of current output

$$
w_{1}^{i} \geq v^{\text {out }}, \forall i .
$$

With two-sided commitment the future promised utility $w_{1}^{i}$ can be unbounded from below as the agent cannot quit (for example if $u(0)=-\infty$ ).

The profit function $\Pi^{C}(w)$ in the first-stage problem (FS) solves the following second-stage problem, defined for any $w \in W$ :

$$
\Pi^{C}(w)=\max _{e,\left\{c^{i}, w^{i}\right\}_{i=1}^{n}} \sum_{i=1}^{n} \pi^{i}(e)\left[y^{i}-c^{i}+r^{-1} \Pi^{C}\left(w^{i}\right)\right]
$$

subject to

$$
\begin{gathered}
\sum_{i=1}^{n} \pi_{e}^{i}(e)\left[u\left(c^{i}\right)+\beta w^{i}\right]-1=0 \\
\sum_{i=1}^{n} \pi^{i}(e)\left[u\left(c^{i}\right)+\beta w^{i}\right]-e=w
\end{gathered}
$$

and, in the case of one-sided commitment, also subject to:

$$
w^{i} \geq v^{\text {out }}, \forall i \text {. }
$$

\footnotetext{
${ }^{22}$ We assume that if the agent leaves his current contract any "promised utility" from the previous insurer is canceled as it is unverifiable.
} 
The first constraint ensures incentive compatibility with the agent's unobserved effort choice. The second constraint, "promise keeping", embodies the commitment ability of the insurer to deliver the promised utility value $w$. The last constraint reflects the lower bound on feasible future promises implied by the agent's inability to commit beyond the current period.

\subsubsection{Auxiliary results}

To prove Proposition 3 we use three auxiliary Lemmas which we prove first.

Lemma 1 For any $\Delta \tau>0$ there exist $\varepsilon^{i}>0, i=1, \ldots, n$ such that: $\sum_{i=1}^{n} \varepsilon^{i}=\Delta \tau$ and $\sum_{i=1}^{n} \pi_{e}^{i}\left[u\left(c^{i}+\varepsilon^{i}\right)-u\left(c^{i}\right)\right]=0$.

Proof. Since $\sum_{i} \pi_{e}^{i}=0$, it is enough to show that we can choose the consumption increments $\varepsilon^{i}$ so that $u\left(c^{i}+\varepsilon^{i}\right)-u\left(c^{i}\right)=b$ where $b$ is some appropriately chosen positive constant. From the strict monotonicity of $u$, a unique solution to this equation in $\varepsilon^{i}$ on $[0, \infty)$ exists and is strictly increasing in $b$, for each $i=1, \ldots n$. Call this solution $\varepsilon^{i}\left(b, c^{i}\right)$. It is a continuous function of $b$ by the continuity and strict concavity of $u$. But then $\sum_{i} \varepsilon^{i}\left(b, c^{i}\right)$ is also continuous and strictly increasing in $b$. Since $\varepsilon^{i}\left(0, c^{i}\right)=0$ for all $c^{i}$ this implies that $\exists b>0$ which solves $\sum_{i} \varepsilon^{i}\left(b, c^{i}\right)=\Delta \tau$ for any $\Delta \tau>0$.

Lemma 1 shows that it is always possible to increase transfers $\tau^{i}$ by appropriate amounts while preserving incentive-compatibility.

To help the further exposition of the proof we reproduce the Markov-perfect insurance problem for any $a \in \mathbb{A}$ (see Section 3.1).

\section{Problem P1}

$$
\mathcal{V}(a)=\max _{e,\left\{\tau^{i}, a^{i}\right\}_{i=1}^{n}} \sum_{i=1}^{n} \pi^{i}(e)\left[u\left(c^{i}\right)+\beta \mathcal{V}\left(a^{i}\right)\right]-e
$$

subject to:

$$
\begin{aligned}
\sum_{i=1}^{n} \pi_{e}^{i}(e)\left[u\left(c^{i}\right)+\beta \mathcal{V}\left(a^{i}\right)\right]-1 & =0 \\
\sum_{i=1}^{n} \pi^{i}(e)\left[y^{i}-\tau^{i}\right] & =0
\end{aligned}
$$

Call P2 the following auxiliary problem defined for any $a \in \mathbb{A}$ and in which $\mathcal{V}(a)$ is the MPE value function from Problem P1. We will show that problems P1 and P2 are mathematically equivalent.

\section{Problem P2}

$$
\Pi(a)=\max _{e,\left\{\tau^{i}, a^{i}\right\}_{i=1}^{n}} \sum_{i=1}^{n} \pi^{i}(e)\left[y^{i}-\tau^{i}+\frac{\Pi\left(a^{i}\right)}{r}\right]
$$

subject to

$$
\begin{aligned}
& \sum_{i=1}^{n} \pi_{e}^{i}(e)\left[u\left(c^{i}\right)+\beta \mathcal{V}\left(a^{i}\right)\right]-1=0 \\
& \sum_{i=1}^{n} \pi^{i}(e)\left[u\left(c^{i}\right)+\beta \mathcal{V}\left(a^{i}\right)\right]-e=\mathcal{V}(a)
\end{aligned}
$$


Lemma 2 (Equivalence of problems P1 and P2) (i) Any solution to problem P1 is a solution to problem P2 for all $a \in \mathbb{A}$. (ii) Any solution to problem P2 is a solution to problem P1 and satisfies $\Pi(a)=0$ for all $a \in \mathbb{A}$.

Proof. For any given $a \in \mathbb{A}$, call $S 1$ a solution to problem $\mathrm{P} 1$ and $S 2$ a solution to problem $\mathrm{P} 2$.

(i) Observe that $S 1$ is feasible for problem P2 (it satisfies both constraints) and, by construction given (ZP), yields $\Pi(a)=0$ for all $a \in \mathbb{A}$. Suppose, however, that $S 1$ is not a solution to problem $\mathrm{P} 2$, that is, the insurer's profits $\Pi(a)$ evaluated at the P2 solution $S 2 \equiv\left\{\tau_{2}^{i}, a_{2}^{i}, e_{2}\right\}$ are strictly positive. This implies that, either in the current or in some future period (omitting time subscripts), $\sum_{i=1}^{n} \pi^{i}\left(e_{2}\right)\left[y^{i}-\tau_{2}^{i}\right]>0$. But then, since $S 2$ satisfies (IC) in problem P1 and achieves the value $\mathcal{V}(a)$ (as does $S 1$ ), we can increase expected transfers in problem P1 (in the appropriate period) starting from allocation $S 2$ until (ZP) is satisfied, while keeping (IC) satisfied (see Lemma 1). Since $u$ is strictly increasing, this transfer increase would yield present value for the agent larger than $\mathcal{V}(a)$ - a contradiction with the assumed optimality of $S 1$ in problem P1.

(ii) Any P2 solution $S 2 \equiv\left\{\tau_{2}^{i}, a_{2}^{i}, e_{2}\right\}$ satisfies (15), that is, $\mathcal{V}(a)=\sum_{i=1}^{n} \pi^{i}\left(e_{2}\right)\left[u\left(c_{2}^{i}\right)+\beta \mathcal{V}\left(a_{2}^{i}\right)\right]-$ $e_{2}$. Hence, $S 2$ achieves the same value of the objective, $\mathcal{V}(a)$ in problem P1 as its solution $S 1$. Clearly, $S 2$ also satisfies (IC) since it is identical to (14). However, could $S 2$ violate the zeroprofit condition (ZP) in P1? Suppose it does. Suppose first that the transfers in $S 2$ were too low relative to output on average, that is, $\sum_{i=1}^{n} \pi^{i}\left(e_{2}\right)\left[y^{i}-\tau_{2}^{i}\right]>0$. But then, in problem P1, we could strictly increase transfers (holding all else constant) starting from allocation $S 2$, while maintaining incentive-compatibility (IC) (see Lemma 1) until (ZP) binds and hence obtain a value for the agent which is strictly larger than the objective function's maximum, $\mathcal{V}(a)$ - a contradiction (recall that $S 2$ attains $\mathcal{V}(a)$ ). Next, suppose the $S 2$ transfers, $\tau_{2}^{i}$ were too high relative to output, that is, $\sum_{i=1}^{n} \pi^{i}\left(e_{2}\right)\left[y^{i}-\tau_{2}^{i}\right]<0$. This would imply that $S 1$ must feature strictly lower expected transfers than $S 2$ since $S 1$ satisfies (ZP) at equality. Therefore, since it attains $\mathcal{V}(a)$ by definition, $S 1$ would satisfy both constraints of problem P2 but yield larger profits than $S 2$ (due to its lower expected transfers). This would contradict the optimality of $S 2$. Therefore, it must be that $S 2$ satisfies constraint (ZP), which means it solves $\mathrm{P} 1$ which implies that its associated profits, $\Pi(a)$ are identically zero for any $a \in \mathbb{A}$.

Lemma 3 Problems $P 1$ and P2 are equivalent to

Problem P3

$$
\hat{\Pi}(a)=\max _{e,\left\{c^{i}, a^{i}\right\}_{i=1}^{n}} \sum_{i=1}^{n} \pi^{i}(e)\left[y^{i}-c^{i}+\frac{\hat{\Pi}\left(a^{i}\right)}{r}\right]
$$

subject to

$$
\begin{aligned}
& \sum_{i=1}^{n} \pi_{e}^{i}(e)\left[u\left(c^{i}\right)+\beta \mathcal{V}\left(a^{i}\right)\right]-1=0 \\
& \sum_{i=1}^{n} \pi^{i}(e)\left[u\left(c^{i}\right)+\beta \mathcal{V}\left(a^{i}\right)\right]-e=\mathcal{V}(a)
\end{aligned}
$$

In addition, $\hat{\Pi}(a)=\Pi(a)$ - ra for all $a \in \mathbb{A}$ where $\Pi(a)$ is defined in problem P2.

Proof. By Lemma 2 problems P1 and P2 are equivalent. Take problem P2 and use that $y^{i}-\tau^{i}=$ $y^{i}-c^{i}+r a-a^{i}$. Defining $\hat{\Pi}(a)=\Pi(a)-r a$, we obtain problem P3. 


\subsubsection{Equivalence between MPE and one-sided commitment}

Proof of Proposition 3. As explained in the main text, the lower bound, $\underline{w}=\mathcal{V}(0)$ of the promised utility set $W^{M P}$ defined in Proposition 3 equals the present value utility of an agent leaving the contract and going to a Markov-perfect insurer with zero assets. This implies $v^{\text {out }}=\mathcal{V}(0)$ in the one-sided commitment problem described in Proposition 3.

Call $\left(w_{1}^{i}, c_{0}^{i}, e_{0}\right),\left\{w^{i}, c^{i}, e\right\}$ the allocation path solving the one-sided commitment problem (FS)(SS) with promised utility set $W^{M P} \equiv[\mathcal{V}(0), \mathcal{V}(\bar{a})]$, starting from some $a_{0} \in \mathbb{A}$. In the proof of Proposition 1 we showed that the agent's value function $\mathcal{V}(a)$ in an MPE is strictly increasing in $a$. Hence, $\mathcal{V}(a)$ is invertible. Consider the second-stage problem SS for any $w \in W^{M P}$. Since $w^{i} \in W^{M P}$ and $\mathcal{V}$ is strictly increasing, we can call $a^{i}=\mathcal{V}^{-1}\left(w^{i}\right)$ and $a=\mathcal{V}^{-1}(w)$. The inverses are well defined since $a^{i} \in \mathbb{A}$ if and only if $w^{i} \in W^{M P}$ and similarly for $a$ and $w$. Since $v^{\text {out }}=\mathcal{V}(0)$, the agent's limited-commitment constraint $w^{i} \geq v^{\text {out }}$ is equivalent to $a^{i} \geq 0$ which is always satisfied.

Call $\tilde{\Pi}\left(a^{i}\right)=\Pi^{C}\left(\mathcal{V}\left(a^{i}\right)\right)$. We can then re-write problem SS as the mathematically equivalent problem

$$
\tilde{\Pi}(a)=\max _{e,\left\{\tau^{i}, a^{i}\right\}_{i=1}^{n}} \sum_{i=1}^{n} \pi^{i}(e)\left[y^{i}-c^{i}+\frac{\tilde{\Pi}\left(a^{i}\right)}{r}\right]
$$

subject to

$$
\begin{aligned}
& \sum_{i=1}^{n} \pi_{e}^{i}(e)\left[u\left(c^{i}\right)+\beta \mathcal{V}\left(a^{i}\right)\right]-1=0 \\
& \sum_{i=1}^{n} \pi^{i}(e)\left[u\left(c^{i}\right)+\beta \mathcal{V}\left(a^{i}\right)\right]-e=\mathcal{V}(a)
\end{aligned}
$$

which is equivalent to problem P3 (and hence, by Lemma 3, to P1) for any $a \in \mathbb{A}$. This implies that the allocation path in problem SS with promised utility choice set $W^{M P}$ and initial value $w=\mathcal{V}(a) \in W^{M P}$ coincides with the MPE allocation path (obtained in problem P1) from initial assets $a$ for any $a \in \mathbb{A}$ and after any output history.

The demonstrated equivalence between problems SS and P3 implies $\tilde{\Pi}(a)=\hat{\Pi}(a)=\Pi(a)-r a$, where $\hat{\Pi}(a)$ and $\Pi(a)$ were defined in Lemma 2 and Lemma 3 and where the second equality follows from Lemma 3 . Since $\Pi(a)=0$ by Lemma 2 , we then obtain

$$
\tilde{\Pi}(a)=\Pi^{C}(\mathcal{V}(a))=-r a \text { for all } a \in \mathbb{A} \text {. }
$$

Consider now the first-stage problem, (FS). Calling $a_{1}^{i}=\mathcal{V}^{-1}\left(w_{1}^{i}\right)$ and using that $\Pi^{C}\left(\mathcal{V}\left(a_{1}^{i}\right)\right)=$ $-r a_{1}^{i}$ as shown above, re-write (FS) as

$$
\max _{e_{0},\left\{c_{0}^{i}, a_{1}^{i}\right\}_{i=1}^{n}} \sum_{i=1}^{n} \pi^{i}\left(e_{0}\right)\left[u\left(c_{0}^{i}\right)+\beta \mathcal{V}\left(a_{1}^{i}\right)\right]-e_{0}
$$

subject to

$$
\begin{aligned}
& \sum_{i=1}^{n} \pi_{e}^{i}\left(e_{0}\right)\left[u\left(c_{0}^{i}\right)+\beta \mathcal{V}\left(a_{1}^{i}\right)\right]-1=0 \\
& \sum_{i=1}^{n} \pi^{i}\left(e_{0}\right)\left[y^{i}+r a_{0}-c_{0}^{i}-a_{1}^{i}\right]=0 .
\end{aligned}
$$


The agent's limited-commitment constraint $w_{1}^{i} \geq v^{\text {out }}$ is satisfied using the same argument as above. Since $y^{i}+r a_{0}-c_{0}^{i}-a_{1}^{i}=y^{i}-\tau_{0}^{i}$, the above problem is mathematically equivalent to the MPE insurance problem $\mathrm{P} 1$ for assets $a_{0}$.

Overall, we have shown that, starting from the same initial assets $a_{0}$, the allocation path implied by the one-sided commitment problem (FS)-(SS) with promised utility set $W^{M P}$ and the MPE allocation path coincide in each time period and for any output history. Therefore, the present values achieved coincide too: $\mathcal{V}^{C}\left(a_{0}\right)=\mathcal{V}\left(a_{0}\right)$ for all $a_{0} \in \mathbb{A}$. 